\title{
Procedimentos Taguchi para Atributos em Horizonte Finito
}

\section{Carlos Takeo Akamine}

Dissertação apresentada ao Instituto de

Matemática e Estatística da

Universidade de São Paulo para

obtenção do grau de Mestre em

Estatística

Área de concentração: Probabilidades

Orientador: Prof. Dr. Wagner de Souza Borges

São Paulo, dezembro de 2000 


\section{Procedimentos Taguchi para Atributos em Horizonte Finito}

Este exemplar corresponde à redação final devidamente corrigida e defendida por Carlos Takeo Akamine e aprovada pela comissão julgadora.

São Paulo, 21 de dezembro de 2000.

Banca examinadora:

- Prof. Dr. Wagner de Souza Borges

- Prof. Dr. Fabio Prates Machado

- Prof. Dra. Linda Lee Ho 


\section{Agradecimentos}

ao Prof. Dr. Wagner de Souza Borges, pela amizade e pela dedicação na orientação do meu trabalho,

aos meus colegas do Instituto de Matemática e Estatística da USP, pela amizade e apoio nas horas de maior dificuldade,

à minha esposa Marina, pela paciência e apoio e ao Arthur, meu filho, por trazer tanta alegria em nossas vidas. 


\section{Resumo}

No presente trabalho estudou-se o procedimento Taguchi "On Line" para atributos em processos de produção de pequenos lotes ("short runs") ou horizonte finito. Dois problemas de produção em horizonte finito, relacionados ao caso em que os itens produzidos são todos conformes quando o processo está sob controle e todos não conformes quando o processo está fora de controle, apresentado por Taguchi et al em 1989, foram analisados. No primeiro, supõe-se que os itens não conformes podem ser retrabalhados para correção. No segundo, os itens não conformes não podem ser corrigidos e são refugados. O objetivo do estudo é encontrar expressões para o custo total de monitoramento que nos permitam otimizar a frequiência de amostragem, minimizando esse custo.

No primeiro problema, foi obtido uma expressão para o custo total de monitoramento que possibilita a obtenção do valor ótimo da frequiência de amostragem. Essa expressão foi ainda utilizada para analisar: o efeito dos custos (de inspeção, de defeituosos e de ajuste do processo); o efeito do número de itens a ser produzido e o efeito do valor da probabilidade do processo sair de controle no valor ótimo da frequiência de amostragem.

No segundo problema, devido à dificuldade na obtenção de uma expressão analítica fechada para o custo total de monitoramento, utilizou-se o método de simulação na análise e na obtenção do valor ótimo da frequiência de amostragem. 


\section{Abstract}

In the present dissertation, Taguchi "On Line" Procedures for Attributes for Short Runs have been studied. Two problems related to the case in which the process fraction defective is 0 or 1 according to whether the process is in control or out of control, respectively, were analyzed. In the first, it is assumed that the defective items produced can be reworked. In the second, reworking is no longer a possibility and it is assumed that the defective items produced are scraped. The main objective is to obtain expressions for the monitoring procedure's total cost that can be used in the optimization of the sampling frequency.

In the first problem, an expression for the monitoring procedure total cost is obtained and used to: minimize the sampling frequency; analyze the effect of changes in the different cost factors; analyze the effect of changes in the lot size and analyze the effect changes in the probability of the process going out of control, over the optimum sampling interval.

In the second problem, due to the difficulty of obtaining an expression for the monitoring procedure total cost in closed form, simulation methods are used to obtain approximate solutions to the sampling frequency optimization problem and to develop sensitivity studies. 
Índice:

pag.

\section{Capítulo 1}

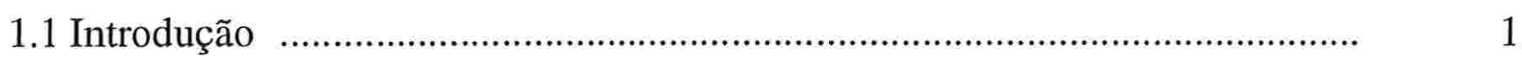

1.2 O Procedimento Taguchi On-Line para Atributos ............................................. 2

1.3 Procedimentos Taguchi para Atributos em Horizonte Finito ........................... 7

\section{Capítulo 2}

2.1 Descrição do problema ……………………………………………………...

2.2 Modelagem ..........................................................................................

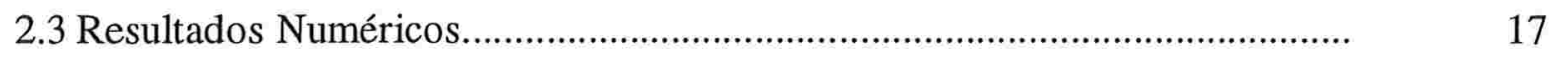

\section{Capítulo 3}

3.1 Descrição do problema ................................................................................

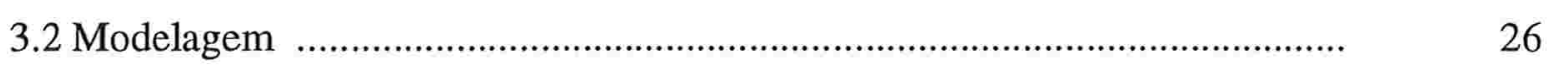

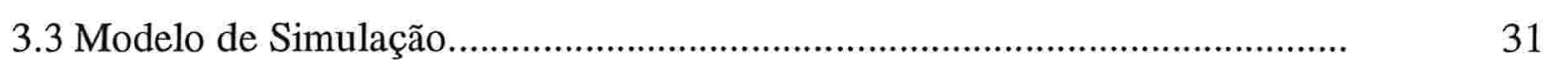

3.4 Resultado Numérico ....................................................................................

\section{Capítulo 4}

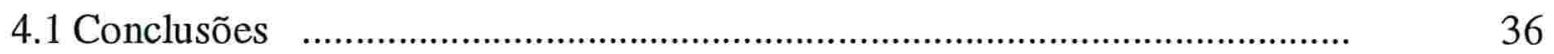

4.2 Sugestões de Pesquisa Futura …………………………………………....... 37

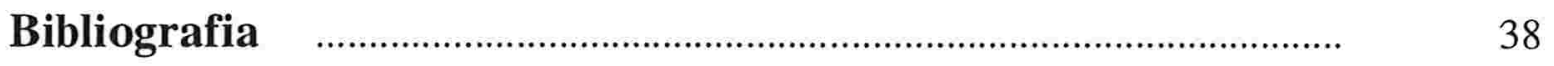

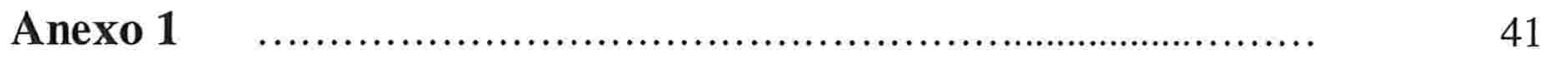

Anexo 2

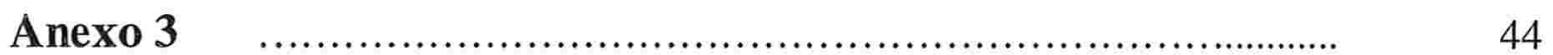




\section{Capítulo 1}

\subsection{Introdução}

Segundo Taguchi, as atividades de controle de qualidade concentram-se em duas áreas principais: aquelas realizadas durante o desenvolvimento do projeto e comumente denominadas de controle de qualidade "off-line", e aquelas realizadas durante a conformação do produto, comumente denominadas controle de qualidade "on-line". O presente trabalho estará voltado à segunda área.

O controle de qualidade "on-line" tem como objetivo avaliar o desempenho do processo de fabricação e as ferramentas de análise mais utilizadas são os gráficos de controle, instrumento criado por Shewart para monitorar a estabilidade de características específicas do processo.

Características de desempenho de qualquer processo de produção, estão sujeitas a variações. As causas da variabilidade, entretanto, são classificadas em: causas comuns, que são efeitos acumulados de um grande número de pequenas causas que pouco influenciam a estabilidade dessas características do processo; e causas especiais, que são efeitos de um pequeno número de causas que influenciam muito a estabilidade dessas características do processo. Um processo é dito sob controle estatístico se estiver sujeito apenas a causas comuns de variação. Ele é dito fora de controle estatístico quando estiver sujeito a causas especiais de variação.

Neste trabalho, enfocaremos unicamente o monitoramento da estabilidade da fração defeituosa de um processo de produção, isto é, da proporção de itens produzidos fora das especificações. O processo estará sob controle estatístico se um item produzido no instante $\mathrm{t}, \mathrm{t} \geq 0$, tiver probabilidade $\mathrm{p}_{\mathrm{t}}=\pi_{\mathrm{o}}$, constante, de não atender as especificações.

$\mathrm{O}$ instrumento clássico de monitoramento, neste caso, é a carta-p. Introduzida por Shewart no início dos anos 30, esta carta nada mais é do que um gráfico dos pontos $\left\{\left(i, \hat{p}_{i}\right) ; i \geq 1\right\}$, em que os $\hat{p}_{i}, \mathrm{i} \geq 1$, são frações defeituosas observadas em amostras do 
processo, selecionadas periodicamente. O gráfico contém ainda três retas paralelas para auxiliar o diagnóstico de perda de controle. São elas a linha central, LC, e os limites inferior e superior de controle, LIC e LSC, respectivamente. Especificamente,

$$
\mathrm{LIC}=\pi_{0}-\mathrm{k} \sqrt{\frac{\pi_{0}\left(1-\pi_{0}\right)}{\mathrm{n}}}, L C=\pi_{0}, L S C=\pi_{0}+k \sqrt{\frac{\pi_{0}\left(1-\pi_{0}\right)}{n}},
$$

em que o tamanho das amostras, $\mathrm{n}$, a amplitude, $\mathrm{k}$, e o intervalo de tempo entre amostras são escolhidos de tal forma que a carta satisfaça a critérios econômicos, estatísticos ou mistos, bem definidos.

Detalhes sobre a construção e operação das cartas-p podem ser encontrados em Montgomery (2000) e Box e Luceño (1998). Nosso interesse específico, estará voltado para uma outra classe de procedimentos para monitorar a estabilidade da fração defeituosa de processos de produção. Mais precisamente, a dos procedimentos Taguchi "on-line” para atributos.

\subsection{O Procedimento Taguchi On-Line para Atributos}

Os primeiros trabalhos de Genichi Taguchi sobre monitoramento da qualidade "online" foram publicados pela primeira vez no livro intitulado "On-line Quality during Production”. Neste livro Taguchi (1981) propôs o seguinte procedimento para monitorar a estabilidade de um processo de produção contínuo:

- o último de cada $\mathrm{m}$ itens produzidos em seqüência é inspecionado

- se o item inspecionado atender às especificações, nenhuma ação sobre o processo é tomada. Caso contrário o processo é interrompido para ajuste, voltando a operar após as correções necessárias.

Taguchi (1981) admite que os itens eram produzidos um a um e que após a produção de um certo número de itens a fração de defeituosa do processo, $\pi$, saltava da 
condição inicial $\pi=\pi_{0}=0$ para $\pi=\pi_{1}>0$. Assim, a observação de um item defeituoso em uma inspeção indicava a fuga da condição de controle ( $\pi=\pi_{0}=0$ constante). Taguchi (1981) admite ainda que após o ajuste a fração defeituosa do processo volta ao valor inicial $\pi=\pi_{0}=0$.

Nesse contexto, Taguchi (1981) sugeriu que o valor de m fosse então escolhido de forma a minimizar o custo médio do procedimento por unidade produzida.

Dois casos foram considerados por Taguchi, o caso 1 , em que $\pi_{1}=1$, e o caso 2 , em que $0<\pi_{1}<1$. Esse trabalho, entretanto, apresentava problemas e foi aperfeiçoado mais tarde por Taguchi, Elsayed e Hsiang (1989) no livro intitulado "Quality Engineering in Production Systems", traduzido para o português em 1990 com o título de "Engenharia da Qualidade em Sistemas de Produção".

No caso 1, Taguchi et al (1989) consideram três fatores de custo

- o custo de um item defeituoso $\left(\mathrm{C}_{\mathrm{d}}\right)$.

- o custo de amostragem e inspeção de um item $\left(\mathrm{C}_{\mathrm{i}}\right)$.

- o custo de ajuste $\left(\mathrm{C}_{\mathrm{a}}\right)$.

Além disso, supõe-se que durante o diagnóstico da peça retirada para inspeção, a produção continua. Se o diagnóstico for de não conformidade, o número de itens produzidos do instante em que o item é amostrado até a parada do processo para ajuste, é designado como atraso e representado por $l$.

A seguinte expressão para o custo esperado do procedimento, por unidade produzida, é então utilizada:

$$
L=\frac{C_{i}}{m}+\left(\frac{m+1}{2}+l\right) \frac{C_{d}}{\bar{u}}+\frac{C_{a}}{\bar{u}}
$$

em que $\bar{u}$ é o número médio de itens produzidos entre ajustes sucessivos.

Observação: Se um item não conforme é inspecionado, uma das situações apresentadas na Figura 1.1 pode ter ocorrido 


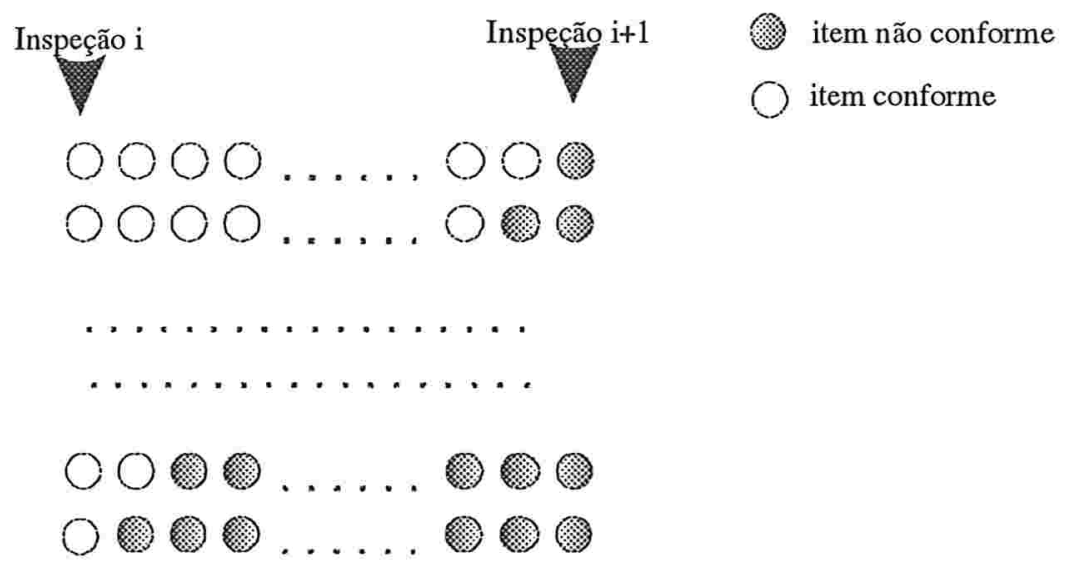

Figura 1.1 Distribuição de produtos não conformes.

Taguchi et al (1989) consideram essas situações como sendo igualmente prováveis. Com isso, o número médio de itens não conformes produzidos entre as duas inspeções é $(\mathrm{m}+1) / 2$.

Resolvendo-se a equação $\frac{d L}{d m}=0$, Taguchi et al (1989) obtém o intervalo ótimo de inspeção, $\mathrm{m}_{\mathrm{o}}$, dado por:

$$
m_{o}=\sqrt{2 \bar{u} \frac{C_{i}}{C_{d}}}
$$

Nesta expressão, ignora-se a dependência de $\bar{u} \mathrm{em} \mathrm{m}$, que será crescente em $\mathrm{m}$. Como $\mathrm{m}_{\mathrm{o}}$ não depende de $l$ nem de $\mathrm{C}_{\mathrm{a}}$, Taguchi et al (1989) sugerem ainda que (1.2) seja aplicada somente se as condições: $\bar{u} \gg l$ e $C_{d} \gg \frac{C_{a}}{\bar{u}}$ forem válidas. Caso isso não ocorra a solução alternativa proposta é substituir $\bar{u}$ por $(\bar{u}+m / 2)$ na expressão (1.1) e obter o valor ótimo de $\mathrm{m}$ a partir da nova expressão para L. Assim,

$L=\frac{C_{i}}{m}+\left(\frac{m+1}{2}\right) \frac{C_{d}}{\bar{u}+m / 2}+\frac{1}{\bar{u}+m / 2}\left(C_{a}+l \cdot C_{d}\right)$ 
e o intervalo ótimo de inspeção é dado por

$$
m_{o}^{*}=\sqrt{\frac{2(\bar{u}+l) C_{i}}{C_{d}-\frac{C_{a}}{\bar{u}}}}
$$

O argumento utilizado por Taguchi et al (1989) para esta substituição é de que o número médio de itens defeituosos produzidos no intervalo em que ocorre a fuga da condição de controle deve ser incorporado em $\bar{u}$. Este argumento é confuso uma vez que $\bar{u}$ já leva em consideração o termo acrescentado.

No caso 2, um novo fator de custo é introduzido e o custo esperado do procedimento por unidade de tempo é expresso por

$$
L=\frac{C_{i}}{m}+(m+1) \frac{C_{D}}{\bar{u}}+\frac{C_{a}}{\bar{u}}+\frac{l C_{d}}{\bar{u}}
$$

Nesta expressão, $\mathrm{C}_{\mathrm{D}}$ representa o custo de um item defeituoso não detectado que chega ao consumidor final. Supondo $C_{D} \gg C_{d}$ e usando o mesmo argumento do caso 1 , o intervalo ótimo de inspeção é dado por

$$
m_{o}=\sqrt{\frac{2(\bar{u}+l) \cdot C_{i}}{2 \cdot C_{D}-\frac{C_{a}}{\bar{u}}}}
$$

Os argumentos que levam a expressão (1.5), entretanto, além de muito confusos, não estão fundamentados em modelo teórico formal. Para corrigir esses problemas, Nayebpour e Woodall (1993) utilizam um argumento da teoria da renovação e calculam o valor de L através da expressão

$$
L=\frac{E(C)}{E(T)},
$$


em que $E(C)$ é o custo esperado do procedimento em um ciclo e $E(T)$ é o número esperado de itens produzidos em um ciclo (intervalo de tempo entre ajustes sucessivos do processo). Assim, a expressão obtida por Nayebpour e Woodall (1993) para L, no caso 1, é

$$
L=\frac{\left(\frac{1}{1-q^{m}}+\text { int eiro }\left(\frac{l}{m}\right)\right) \cdot C_{i}+\left(m-\frac{q}{1-q}+\frac{m q^{m}}{1-q^{m}}+l\right) \cdot C_{d}+C_{a}}{\frac{m}{1-q^{m}}+l}
$$

Observação: o valor de L na expressão (1.8) representa, na realidade, o custo médio assintótico do procedimento por unidade produzida.

Para o caso 2, as expressões para $\mathrm{E}(\mathrm{C})$ e $\mathrm{E}(\mathrm{T})$ obtidas por Nayebpour e Woodall (1993) são

$$
E(C)=E\left(L_{D}\right)+\left(\frac{\left(\frac{m}{1-q^{m}}+\frac{m(1-\pi)}{\pi}\right)}{m}+\operatorname{int} \operatorname{eiro}\left(\frac{l}{m}\right)\right) \cdot C_{i}+C_{a},
$$

em que

$$
E\left(L_{D}\right)=\left(\left(m-\frac{q}{1-q}+\frac{m q^{m}}{1-q^{m}}\right) \cdot \pi^{2}+m \pi(1-\pi)+l \pi\right) \cdot C_{d}+\left(\left(m-\frac{q}{1-q}+\frac{m q^{m}}{1-q^{m}}\right) \cdot \pi(1-\pi)+m(1-\pi)^{2}\right) \cdot C_{D}
$$

$\mathrm{e}$

$$
E(T)=\frac{m}{1-q^{m}}+\frac{m(1-\pi)}{\pi}+l
$$


Nas expressões obtidas por Nayebpour e Woodall (1993), q, $0<\mathrm{q}<1$, é o parâmetro da distribuição geométrica que descreve o número de itens conformes produzidos em um ciclo, um elemento não presente nos modelos sugeridos por Taguchi et al (1989).

Devido à complexidade das expressões obtidas por Nayebpour e Woodall (1993) para L, os valores ótimos de $\mathrm{m}$, tanto no caso 1 quanto no caso 2 só podem ser obtidos por busca direta. Nayebpour e Woodall (1993) observam ainda que os valores ótimos de m obtidos através das relações (1.4) e (1.8) dão resultados muito próximos, apesar das considerações e aproximações "estranhas" feitas por Taguchi et al (1989). Entretanto, os valores ótimos de m obtidos através das expressões (1.6) e (1.9 e 1.11) são bem distintos. No caso 2, portanto, recomenda-se o uso da relação (1.9 e 1.11) para a obtenção do valor ótimo de $\mathrm{m}$.

As estratégias propostas tanto por Taguchi et al (1989) quanto por Nayebopour e Woodall (1993), são semelhantes às propostas por Gibra (1978), Montgomery, Heikes e Mance (1975) e Willians, Looney e Peters (1985) para cartas p economicamente planejadas.

\subsection{Procedimentos Taguchi para Atributos em Horizonte Finito}

As estratégias de monitoramento de qualidade apresentadas anteriormente, pressupõem que os processos operarão continuamente ou em horizonte de tempo infinito. Muitas vezes, entretanto, as empresas trabalham com baixos volumes de produção, isto é, em pequenos lotes (short runs). O conceito de pequeno lote, entretanto, é bastante relativo e seu tamanho pode variar de algumas dezenas a até milhares de peças dependendo do tipo de empresa, do tipo de peça e da complexidade do processo de produção. Existem processos que podem durar um dia para produzir algumas dezenas de peças enquanto outros, podem produzir milhares de peças num único dia.

Assim, é de interesse o estudo de procedimentos Taguchi para monitoramento da fração defeituosa em processos de produção limitada ou em horizonte finito. Isto será desenvolvido nos dois próximos capítulos. Nesse estudo iremos tratar de dois problemas de prođução em horizonte finito, relacionados ao caso 1 do procedimento descrito na seção 
anterior. O primeiro, analisado no capítulo 2, em que os itens não conformes podem ser retrabalhados para correção. Neste problema, a quantidade de itens produzidos será constante uma vez que as peças não conformes produzidas serão corrigidas no retrabalho. Dessa forma, o número de inspeções programadas será também constante e dependerá do valor de $\mathrm{m}$. As variáveis aleatórias básicas nesse problema serão: o número de peças não conformes e o número de inspeções retrospectivas para detecção dos itens não conformes produzidos.

O segundo, analisado no capítulo 3 , os itens não podem ser corrigidos e são refugados. Neste problema, a quantidade de itens produzidos será variável pois os itens não conformes produzidos serão tratados como refugo, devendo, portanto, ser repostos.

O objetivo desse estudo é encontrar relações que nos permitam otimizar a freqüência de amostragem, minimizando os custos de monitoramento da qualidade.

No capítulo 2, desenvolveu-se uma relação que possibilita a obtenção do valor ótimo da frequiência de amostragem e, dessa relação, analisou-se o efeito dos custos (de inspeção, de defeituosos e de ajuste do processo), o efeito do número de itens a ser produzido e o efeito do valor da probabilidade do processo sair de controle no valor ótimo da frequiência de amostragem.

No capítulo 3, devido a dificuldade na obtenção da relação que otimiza a freqüência de amostragem, utilizou-se a simulação do processo de produção para a obtenção do valor ótimo da frequiência de amostragem. 


\section{Capítulo 2}

Muitas indústrias, como de confecção de roupas e de calçados, produzem peças que podem ser retrabalhados quando apresentar defeitos de fabricação. Neste capítulo será tratada a questão do monitoramento da qualidade na linha através do procedimento Taguchi, para sistemas de produção limitada em que os itens não conformes podem ser retrabalhados para correção.

\subsection{Descrição do problema}

Existem vários processos em que os itens não conformes produzidos podem ser retrabalhados para correção. Para identificá-los, o seguinte procedimento é utilizado:

- o último de cada m itens produzidos em seqüência é inspecionado;

- se o item inspecionado é conforme, o processo de produção continua em operação. Caso contrário, interrompe-se o processo de produção para ajuste e os itens não-conformes produzidos são identificados por inspeção retrospectiva.

Supõe-se que, enquanto ajustado, o processo produz apenas itens conformes e que ao perder o ajuste todos os itens produzidos serão não-conformes. Assim, a busca retrospectiva descrita acima irá limitar-se às últimas m unidades produzidas. Supõe-se também que o número de unidades a ser produzido é $\mathrm{T}$, um número finito fixado.

A Figura 2.1 descreve o procedimento utilizado. A quantidade de itens a ser produzida será constante e igual a $\mathrm{T}$, pois todos os itens não conformes serão retrabalhados para correção.

O objetivo neste caso é encontrar o intervalo ótimo de inspeção, isto é, o valor de m que minimiza o custo médio total do procedimento. Os fatores de custos associados ao procedimento são:

- o custo da inspeção programada de $\mathrm{m}$ em m itens $\left(\mathrm{C}_{\text {insp }}\right)$;

- o custo do retrabalho $\left(\mathrm{C}_{\text {retrab }}\right)$;

- o custo de ajuste do processo $\left(\mathrm{C}_{\text {ajuste }}\right)$. 


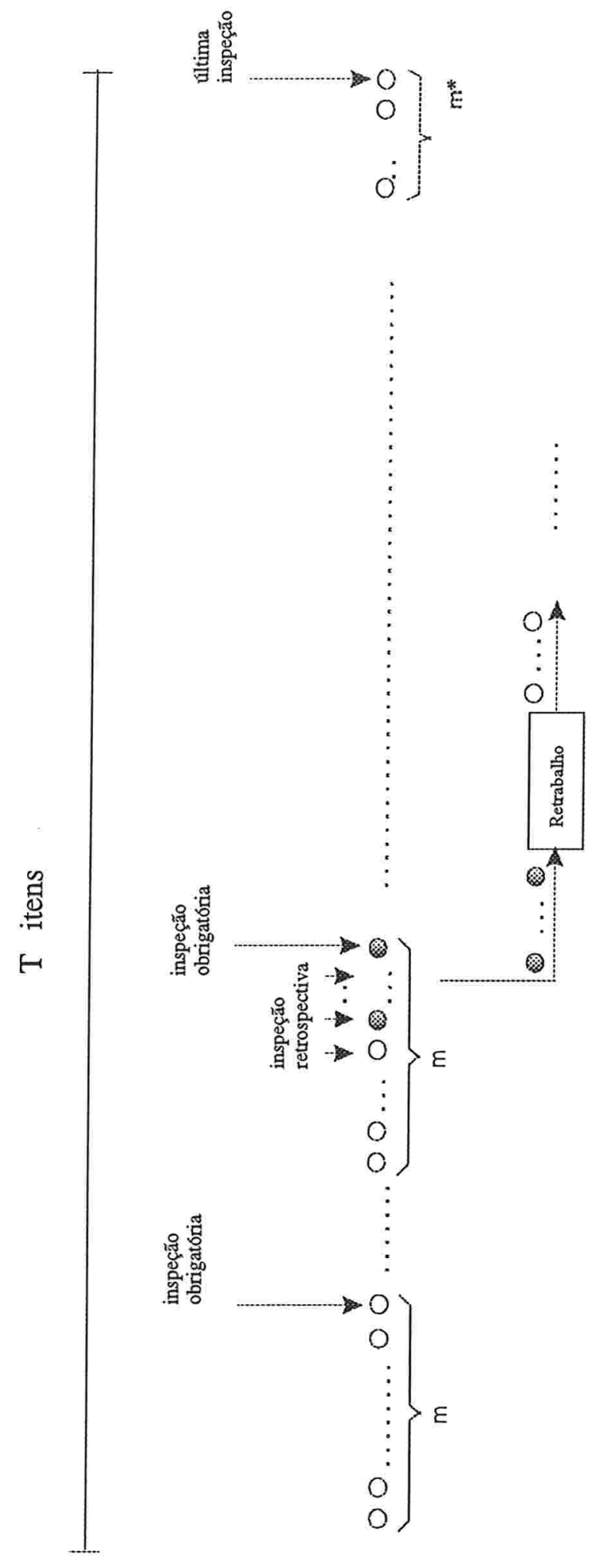

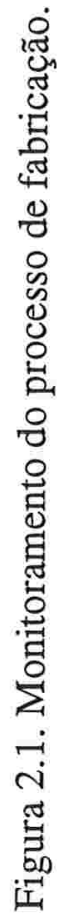


O último intervalo de inspeção, representado por $m^{*}$ na Figura 2.1, será menor ou igual a $m$, isto é, se T for um múltiplo de m então $m^{*}$ será igual a $m$, caso contrário será menor.

\subsection{Modelagem}

Para o problema em questão, as seguintes considerações são feitas:

- quando o processo está sob controle todos os itens produzidos são conformes e quando fora de controle todos os itens produzidos são não conformes.

- os itens não conformes identificados serão retrabalhados e transformados em itens conformes.

- a inspeção é instantânea, isto é, nenhum item novo é produzido durante a inspeção.

- após a inspeção de um item não conforme o processo é instantaneamente interrompido para ajuste e inicia-se a inspeção retrospectiva para a identificação de todos os itens não conformes produzidos.

- a inspeção é feita num único item.

- O número de itens conformes produzidos até a perda de ajuste do processo tem distribuição geométrica com parâmetro $\mathrm{p}, 0<\mathrm{p}<1$.

O número de inspeções programadas será, portanto, igual ao menor inteiro maior ou igual a $\mathrm{T} / \mathrm{m}$, representado por $] \frac{T}{m}\left[\right.$. Assim, o valor de $\mathrm{m}^{*}$ será dado pela seguinte relação:

$$
m^{*}=T-(] \frac{T}{m}[-1) \cdot m
$$

Chamaremos de bloco $\mathrm{j}$, que será representado por $\left.\mathrm{B}_{\mathrm{j}}, 1 \leq \mathrm{j} \leq\right] \frac{T}{m}[$, o conjunto de itens produzidos após a inspeção (j-1), até a inspeção j. Os blocos $B_{j-1}, B_{j}, B_{j+1}$ estão ilustrados na Figura 2.2. Observe que se a inspeção programada $\mathrm{em}_{\mathrm{j}}$ revelar que o item é 
conforme, não haverá inspeção retrospectiva. Caso contrário, devemos inspecionar retrospectivamente os itens produzidos até encontrarmos um item não defeituoso. Assim, teremos identificado todos os itens não conformes em $B_{\mathrm{j}}$. Um caso particular ocorre quando todos os itens em $B_{\mathrm{j}}$ são não conformes. Nesse caso a inspeção retrospectiva termina quando examinarmos todos os itens do bloco.

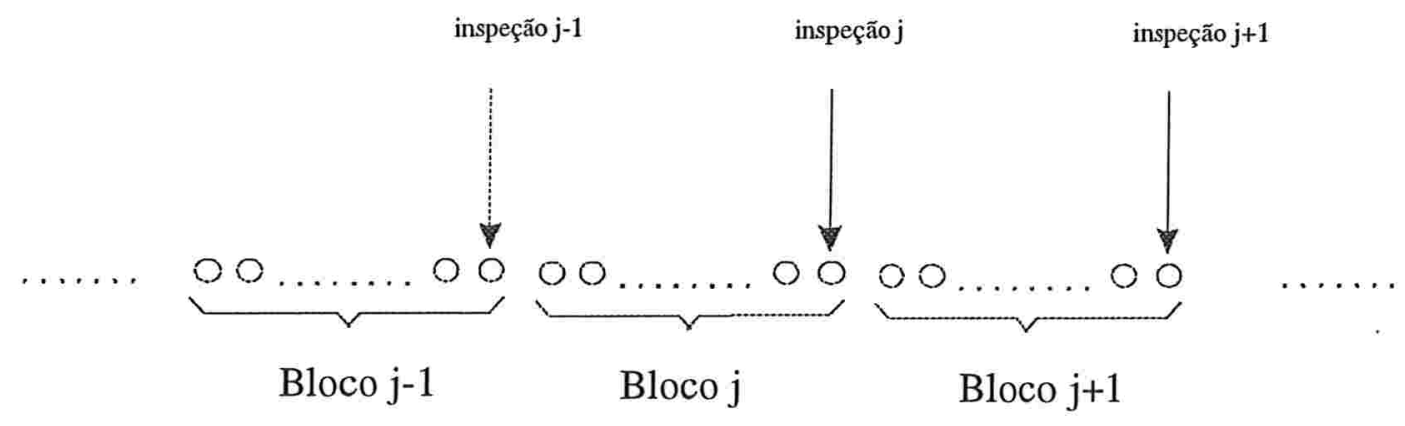

Figura 2.2 Ilustração dos blocos $\mathrm{B}_{\mathrm{j}-1}, \mathrm{~B}_{\mathrm{j}}, \mathrm{B}_{\mathrm{j}+1}$.

Representando por $\mathrm{Mj}$ e $\mathrm{D}_{\mathrm{j}}$ as variáveis aleatórias, número de inspeções e número de itens não conformes em $B_{j}$, respectivamente, o custo total esperado de produção de $T$ unidades é dado por:

$C=\sum_{j=1}^{\frac{T}{m}}\left(C_{\text {insp }} \cdot E\left(M_{j}\right)+C_{\text {retrab }} \cdot E\left(D_{j}\right)\right)+\sum_{j=1}^{]^{\frac{T}{m}-1}[} C_{\text {ajuste }} \cdot E\left(1\left\{D_{j}>0\right\}\right)$.

Observação: Note que não há a necessidade de ajuste no último bloco.

Levando-se em conta a falta de memória do mecanismo de falha do processo, as funções de probabilidade das variáveis aleatórias $\mathrm{Mj}$ e Dj são dadas por

$$
P\left(M_{j}=i\right)= \begin{cases}(1-p)^{m} & i=1 \\ (1-p)^{m-i+1} \cdot p & i=2, \ldots, m-1 \\ (1-p) \cdot p+p & i=m \\ 0 & \text { caso contrário }\end{cases}
$$

e 
$P\left(D_{j}=i\right)= \begin{cases}(1-p)^{m} & i=0 \\ (1-p)^{m-i} \cdot p & i=1, \ldots, m \\ 0 & \text { caso contrário }\end{cases}$

para $1 \leq \mathrm{j}<] \frac{T}{m}[$.

Observe que se $\mathrm{X}$ é o número de itens conformes produzidos até a perda de ajuste do processo, teremos:

$$
P\left\{M_{j}=1\right\}=P\left\{D_{j}=0\right\}=P\{X \geq j m \mid X \geq(j-1) m\}=(1-p)^{m}
$$

Para $1<\mathrm{i}<\mathrm{m}$,

$$
\begin{aligned}
P\left\{M_{j}=i\right\} & =P\left\{D_{j}=i-1\right\}=P\{X=(j-1) m+(m-i+1) \mid X \geq(j-1) m\} \\
& =\frac{p(1-p)^{(j-1) m+(m-i+1)}}{(1-p)^{(j-1) m}}=p \cdot(1-p)^{m-i+1}
\end{aligned}
$$

Como referência, a Figura 2.3 representa o evento $\left\{M_{j}=i\right\}$.

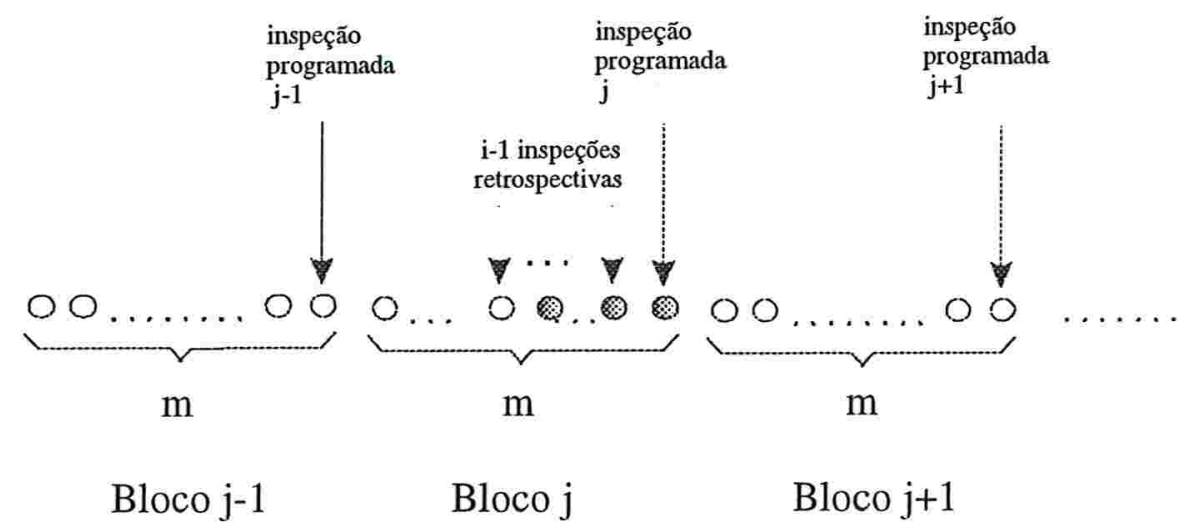

Figura 2.3 Ilustração do caso de i-1 inspeções retrospectivas em $\mathrm{B}_{\mathrm{j}}$. 
O evento $\left\{M_{j}=m\right\}$ ocorre se uma das duas situações indicadas na Figura 2.4 se configurar. Assim,

$$
\begin{aligned}
P\left\{M_{j}=m\right\} & =P\{(j-1) m \leq X \leq(j-1) m+1 \mid X \geq(j-1) m\} \\
& =\frac{p(1-p)^{(j-1) m}+p(1-p)^{(j-1) m+1}}{(1-p)^{(j-1) m}}=p+p(1-p)
\end{aligned}
$$

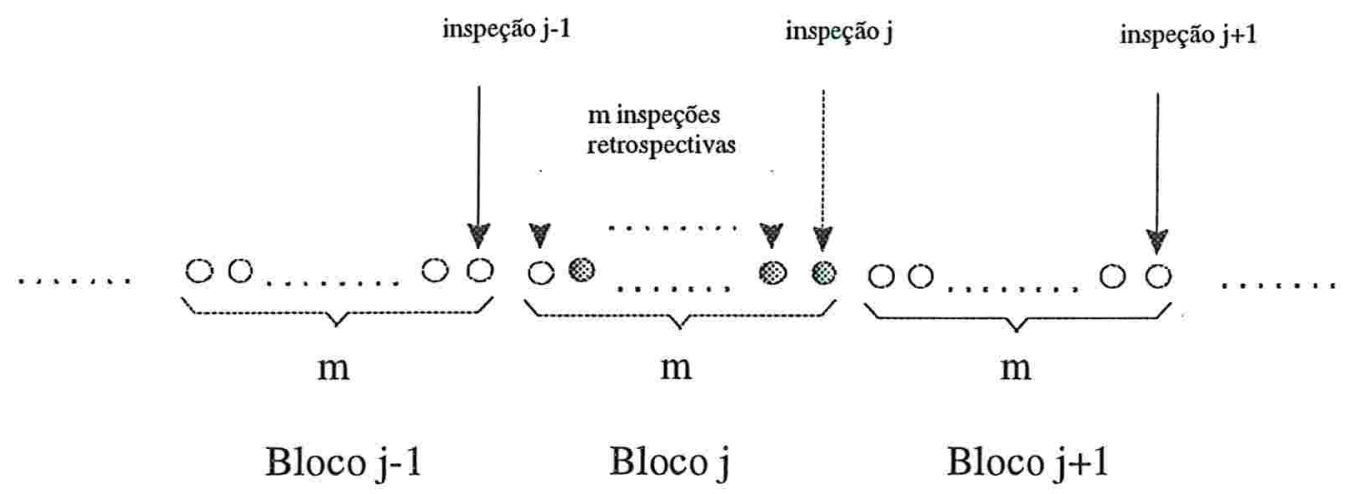

a) m-1 não conformes

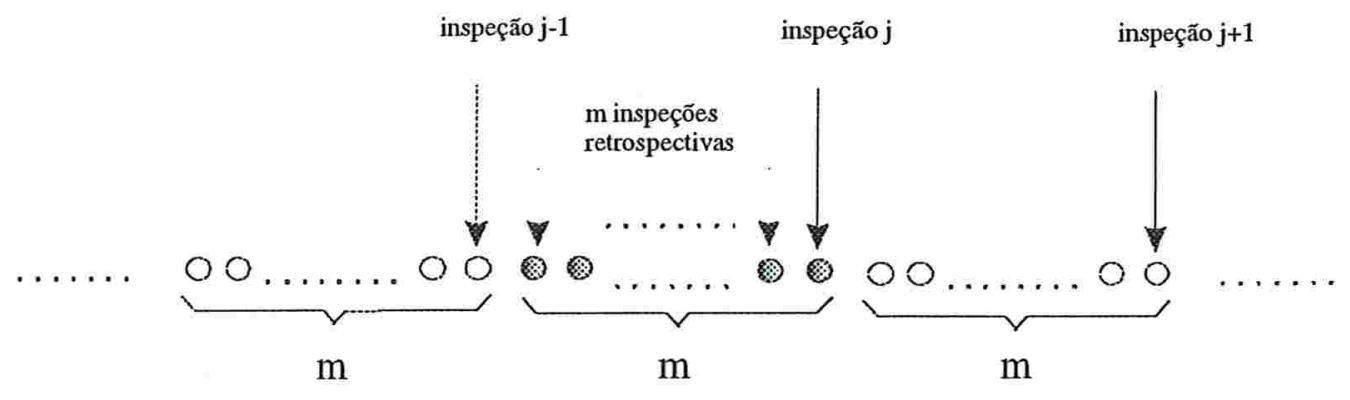

$$
\text { Bloco j-1 Bloco j Bloco } \mathrm{j}+1
$$

b) m não conformes

Figura 2.4 Ilustração do caso de $m$ inspeções retrospectivas em $B_{j}$.a) para $m-1$ não conformes b) para m não conformes. 
Assim, as esperanças de $\mathrm{M}_{\mathrm{j}}$ e $\mathrm{D}_{\mathrm{j}}$ serão dadas por:

$$
\begin{aligned}
E\left(M_{j}\right) & =\sum_{i=1}^{m} i \cdot P\left(M_{j}=i\right)=(1-p)^{m}+\sum_{i=2}^{m}\left(i \cdot(1-p)^{m-i+1} \cdot p\right)+m p \\
& =(1-p)^{m}+m p+p \cdot(1-p)^{m+1} \sum_{i=2}^{m} i \cdot(1-p)^{-i} \\
& =(1-p)^{m}+m p+p \cdot(1-p)^{m+1} \cdot\left[\frac{(m p-(1-p))-(1-p)^{m-2+1} \cdot(2 p-1)}{(1-p)^{m} \cdot p^{2}}\right] \\
& =(1-p)^{m}+m p+\frac{(1-p)(m p-1+p)-(1-p)^{m} \cdot(2 p-1)}{p} \\
& =\frac{(1-p)^{m+1}+\left(m p-1+2 p-p^{2}\right)}{p}
\end{aligned}
$$

Observação: Se o valor de p é igual a 1, isto é, todo item após a perda de controle será não conforme com probabilidade 1 , o valor esperado de $M_{j}$ será $m$. Por outro lado, quando o valor de p tende a zero, $\lim _{p \rightarrow 0} E\left(M_{j}\right)=1$.

$$
\begin{aligned}
E\left(D_{j}\right) & =\sum_{i=0}^{m} i \cdot P\left(D_{j}=i\right)=\sum_{i=1}^{m-1} i \cdot(1-p)^{m-1} \cdot p+m p=m p+p(1-p)^{m} \cdot \sum_{i=1}^{m-1} i \cdot(1-p)^{-i} \\
& =m p+p(1-p)^{m} \cdot\left[\frac{((m-1) \cdot p-(1-p))-(1-p)^{m-1}}{(1-p)^{m-1} \cdot p^{2}} \cdot(p-1)\right] \\
& =m p+p(1-p)^{m} \cdot \frac{(m p-1)+(1-p)^{m}}{p}=m p+(1-p) \cdot \frac{(m p-1)+(1-p)^{m}}{p} \\
& =m p+\frac{(1-p)^{m+1}}{p}+\frac{1-p}{p}+\frac{m p-m p^{2}-1+p}{p} \\
& =m+\frac{(1-p)^{m+1}-(1-p)}{p}
\end{aligned}
$$

Observação: Se o valor de $p$ é igual a 1, isto é, todo item após a perda de controle será não conforme com probabilidade 1 , o valor esperado de $D_{j}$ será $m$. Por outro lado, quando o valor de p tende a zero, $\lim _{p \rightarrow 0} E\left(D_{j}\right)=0$. 
Como no último bloco $\left(\mathrm{B}_{\mathrm{j}}, j=\right] \frac{T}{m}[)$, o número de itens é $m^{*}$, os valores esperados para o número de itens defeituosos e o número de inspeções serão análogos aos dos blocos anteriores. Especificamente,

$$
\begin{aligned}
& E\left(M_{] \frac{T}{m}[}\right)=\frac{(1-p)^{m^{*}+1}+\left(m^{*} p-1+2 p-p^{2}\right)}{p} \\
& E\left(D_{] \frac{T}{m}[}\right)=m^{*}+\frac{(1-p)^{m^{*}+1}-(1-p)}{p}
\end{aligned}
$$

Assim, o custo total esperado do procedimento será

$$
\begin{aligned}
\mathrm{C}= & C_{\text {retrab. }} \cdot \sum_{i=1}^{\frac{T}{m}[-1}\left(m+\frac{(1-p)^{m+1}-(1-p)}{p}\right)+C_{\text {retrab. }} \cdot\left(m^{*}+\frac{(1-p)^{m^{*}+1}-(1-p)}{p}\right) \\
C_{\text {insp. }} \cdot & \sum_{i=1}^{\frac{T}{m}[-1}\left(\frac{(1-p)^{m+1}+\left(m p-1+2 p-p^{2}\right)}{p}\right)+C_{\text {insp. }} \cdot\left(\frac{(1-p)^{m^{*}+1}+\left(m^{*} p-1+2 p-p^{2}\right)}{p}\right) \\
& C_{\text {ajuste }} \cdot \sum_{i=1}^{\frac{T}{m}[-1}\left(1-(1-p)^{m}\right)
\end{aligned}
$$

Observe que a complexidade da expressão de $\mathrm{C}$ torna inviável a determinação do valor ótimo de $\mathrm{m}$ através de métodos analíticos. Portanto, o valor ótimo de $m$ será obtido através do cálculo do custo esperado total variando $m$ de 1 a $T$. Esses cálculos foram realizados no software S-Plus e o programa para esses cálculos encontra-se no Anexo 1. Esse programa calcula os valores do custo total esperado, mostra um gráfico e encontra $m$ que minimiza o custo esperado total. 


\subsection{Exemplos Numéricos}

Neste item estudaremos dois exemplos numéricos. No primeiro, o custo de retrabalho será relativamente baixo e no segundo alto. Além disso, iremos avaliar o efeito de cada custo no valor ótimo de m.

Exemplo 1: Considerando $T=500$, custos de monitoramento dados por:

$\mathrm{C}_{\text {insp }}=\$ 10 ; \mathrm{C}_{\text {retrab }}=\$ 50 ; \mathrm{C}_{\mathrm{ajuste}}=\$ 400$

e valores de $p$ iguais a 0,$3 ; 0,1 ; 0,01$ e 0,001 . Os valores de $m$ que minimizam o custo total esperado para cada um dos quatro casos são dados na Tabela 2.1. Na Figura 2.5 apresentamos os gráficos dos custo total esperado em função de $m$.
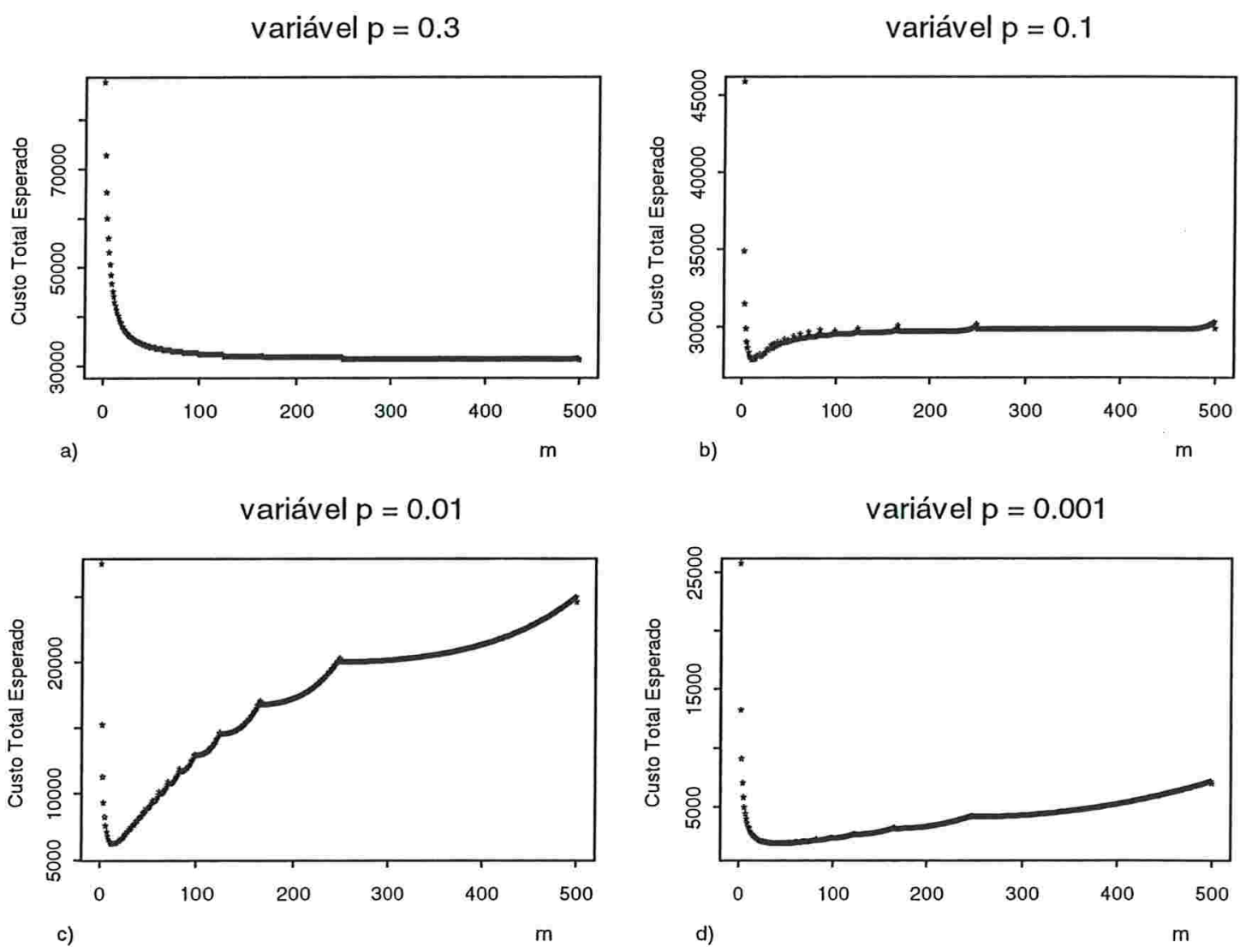

Figura 2.5 Gráfico de Custo Total Esperado em função de m para vários valores de p. a) $p=0,3$ b) $p=0,1$ c) $p=0,01$ d) $p=0,001$. 
Tabela 2.1: Tabela do valor ótimo de m para vários valores de p.

\begin{tabular}{|c|c|c|c|}
\hline Caso & $\mathrm{p}$ & Custo Total Esperado & m ótimo \\
\hline $\mathrm{a}$ & 0,3 & 29895.00 & 500 \\
\hline $\mathrm{b}$ & 0,1 & 27430.22 & 10 \\
\hline $\mathrm{b}$ & 0,01 & 5763.843 & 14 \\
\hline $\mathrm{d}$ & 0,001 & 1412.294 & 42 \\
\hline
\end{tabular}

A descontinuidade nos gráficos ocorre devido à mudança dos valores de $m * \mathrm{em}$ função de m. Dos quatro casos analisados, observamos que existe um ponto de mínimo exceto quando $\mathrm{p}=0,3$. Na Figura 2.5.a temos o gráfico decrescente e o valor de $m$ que minimiza o custo total será $\mathrm{m}=\mathrm{T}$. Nesse caso, vale mais a pena realizar uma inspeção no final do processo e encontrar os não conformes e retrabalhá-los, sem a necessidade de nenhum ajuste no processo.

Usando os mesmos valores de custos e do tamanho do lote, $\mathrm{T}$, calculamos ainda o valor de $m$ que minimiza o custo esperado total para vários valores de p. Esses cálculos foram obtidos através de um programa desenvolvido para o software S-Plus que se encontra no Anexo 2. O resultado encontra-se na Tabela 2.2 e o gráfico desses resultados na Figura 2.6. 
Tabela 2.2 Tabela do valor ótimo de m em função de p.

\begin{tabular}{|c|c|}
\hline $\mathrm{P}$ & m ótimo \\
\hline 0.000001 & 500 \\
\hline 0.000010 & 500 \\
\hline 0.000050 & 167 \\
\hline 0.000090 & 125 \\
\hline 0.000100 & 125 \\
\hline 0.000300 & 72 \\
\hline 0.000700 & 50 \\
\hline 0.001000 & 42 \\
\hline 0.003000 & 25 \\
\hline 0.010000 & 14 \\
\hline 0.030000 & 10 \\
\hline 0.050000 & 8 \\
\hline 0.090000 & 10 \\
\hline 0.100000 & 10 \\
\hline 0.105000 & 14 \\
\hline 0.110000 & 20 \\
\hline 0.115000 & 25 \\
\hline 0.118000 & 36 \\
\hline 0.119000 & 72 \\
\hline 0.119030 & 84 \\
\hline 0.119032 & 100 \\
\hline 0.119036 & 125 \\
\hline 0.119037 & 500 \\
\hline 0.300000 & 500 \\
\hline 0.500000 & 500 \\
\hline & \\
\hline & \\
\hline
\end{tabular}


Gráfico de $\mathrm{m}($ minimo $) \times p$

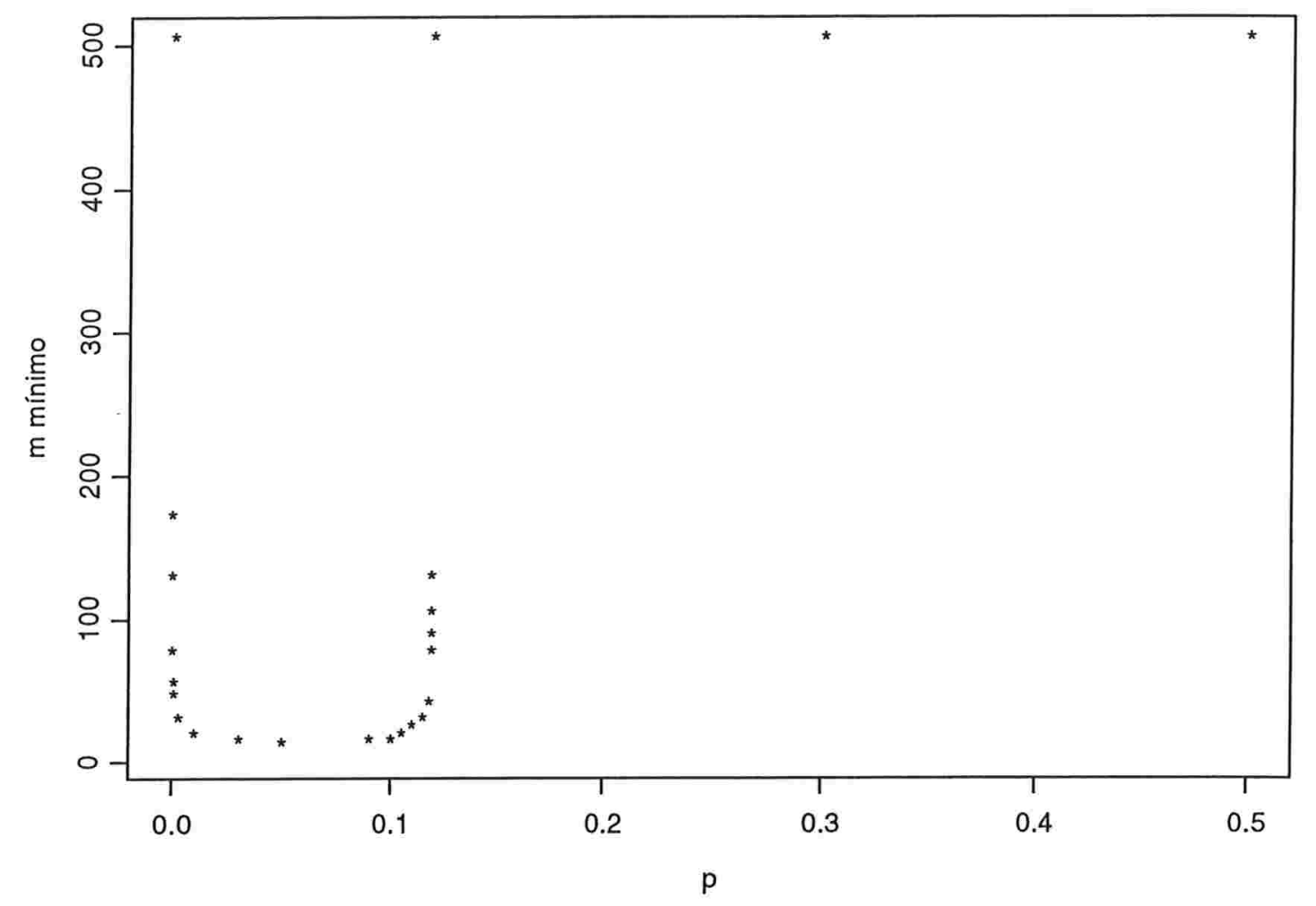

Figura 2.6 Gráfico do valor de m que minimiza o custo total esperado para vários valores de $\mathrm{p}$.

Observe que o valor ótimo de $\mathrm{m}$ decresce quando $\mathrm{p}$ está próximo a 0,00001 até aproximadamente 0,05 e depois cresce até aproximadamente 0,12 depois o valor de $\mathrm{m}$ estaciona em 500.

Exemplo 2: Considere agora a situação em que o custo de ajuste é alto e $\mathrm{p}=0,000339$

$\mathrm{C}_{\text {insp. }}=\$ 150$;

$\mathrm{C}_{\text {retrab. }}=\$ 5$;

$\mathrm{C}_{\text {ajuste }}=\$ 4000$. 
Esses dados foram utilizados por Berry (1974) e Nayebpour e Woodall (1993) para ilustrar a determinação de intervalos ótimos de inspeção. Como nessas aplicações não há custo de retrabalho, consideramos que o mesmo seja igual ao custo de um item defeituoso utilizado nas referências citadas.

Foram avaliados quatro valores de T: 100, 1000, 10000 e 50000.

Os valores de m que minimizam o custo total esperado para cada um dos quatro casos são dados na Tabela 2.3. Quando $\mathrm{T}=100$, o valor ótimo de $m$ é uma única inspeção programada e conseqüentemente não haverá nenhum ajuste. Para os outros valores de $\mathrm{T}$ o valor ótimo de m fica estável em 77 ou 76. Para confirmar esta estabilidade foi feito mais um cálculo com $\mathrm{T}=100000$ e o resultado obtido foi de m ótimo igual a 76. A Figura 2.7 mostra o comportamento do custo total esperado em função de $\mathrm{m}$ para vários valores de $\mathrm{T}$.

Tabela 2.3 Tabela de resultados do m ótimo em função de T.

\begin{tabular}{|c|c|c|c|}
\hline Caso & $\mathrm{T}$ & Custo Total Esperado & m ótimo \\
\hline 1 & 100 & 412.35 & 100 \\
\hline 2 & 1000 & 5216.08 & 77 \\
\hline 3 & 10000 & 53090.84 & 77 \\
\hline 4 & 50000 & 265918.10 & 76 \\
\hline
\end{tabular}


Total $=100$
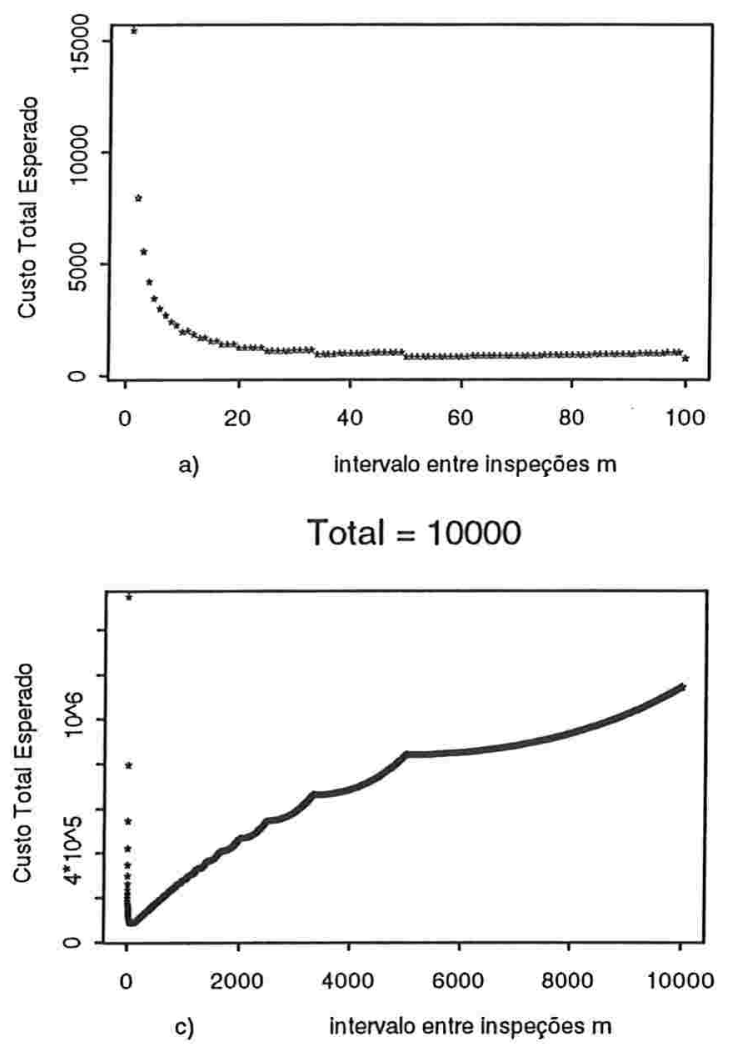

Total $=1000$

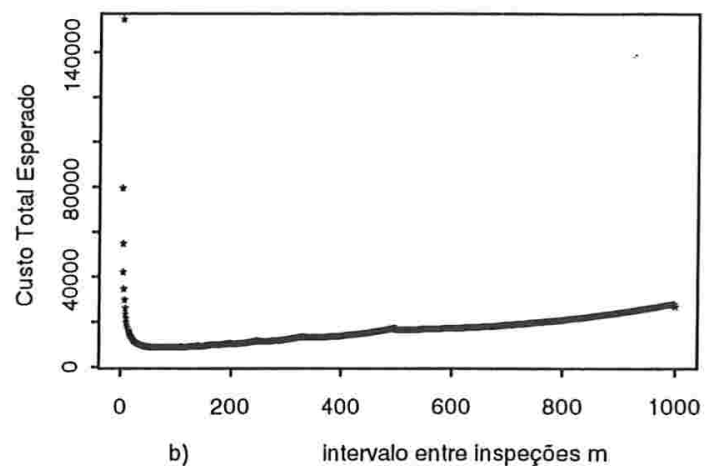

Total $=50000$

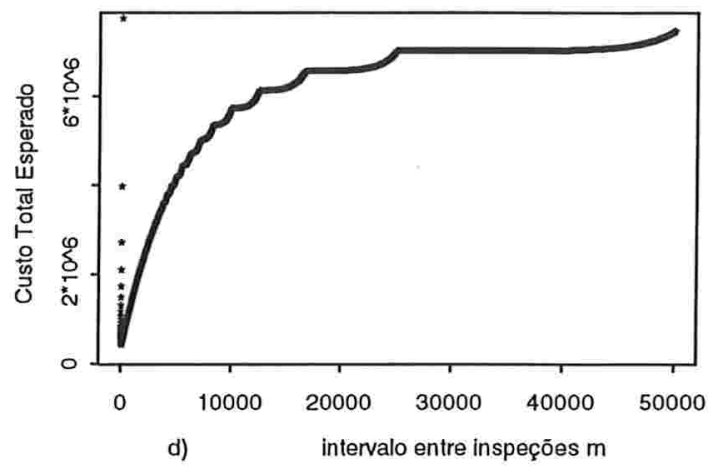

Figura 2.7 Gráficos do custo total esperado em função de $m$.

Uma vez que o procedimento utilizado neste trabalho difere substancialmente do procedimento utilizado por Nayebpour e Woodall (1993), particularmente no que diz respeito às inspeções retrospectivas, optou-se não fazer uma comparação dos dois resultados obtidos.

Para avaliar a influência de cada custo no valor ótimo de $m$, consideramos os custos relacionados na Tabela 2.4. Combinações desses valores foram também utilizadas por Nayebpour e Woodall (1993) na avaliação de desempenho econômico do procedimento em horizonte infinito. Outros dados são: $\mathrm{p}=0,01$ e $\mathrm{T}=1000$. 
Tabela 2.4 Custos de inspeção, defeituosos e de ajuste avaliados.

\begin{tabular}{|l|c|c|}
\hline \multirow{2}{*}{ Custos de } & - & + \\
& -15 & 150 \\
\hline Inspeção (\$) & 15 & 50 \\
\hline Defeituoso (\$) & 0,5 & 4000 \\
\hline Ajuste (\$) & 400 & \\
\hline
\end{tabular}

Os valores ótimos de $m$ para todas as combinações dos níveis dos custos estão na Tabela 2.5 e na Figura 2.8.

Tabela 2. 5 Valores ótimos de m para todas combinações de custos.

\begin{tabular}{|c|c|c|c|c|}
\cline { 2 - 5 } \multicolumn{1}{c|}{} & \multicolumn{3}{c|}{ Custos } & \multicolumn{1}{c}{} \\
\cline { 2 - 5 } \multicolumn{1}{c|}{} & Inspeção & Defeituoso & Ajuste & m ótimo \\
\hline 1 & - & - & - & 17 \\
\hline 2 & + & - & - & 7 \\
\hline 3 & - & + & - & 13 \\
\hline 4 & + & + & - & 1000 \\
\hline 5 & - & - & + & 20 \\
\hline 6 & + & + & + & 13 \\
\hline 7 & - & + & + & 13 \\
\hline 8 & + & & + & \\
\hline
\end{tabular}




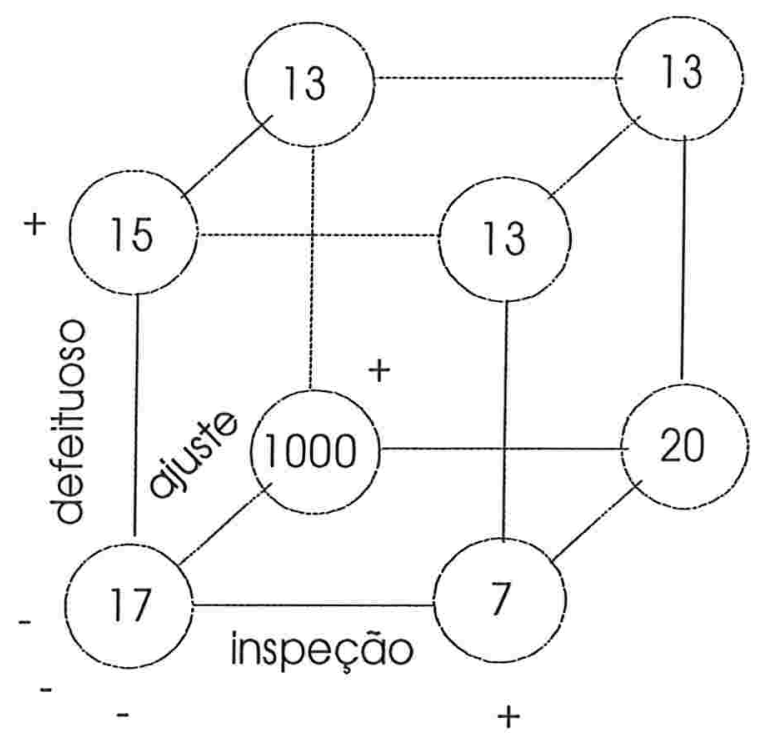

Figura 2.8 Valores ótimos de m para todas as combinações dos custos.

A Figura 2.8 mostra que o aumento do custo de inspeção diminui o valor ótimo de $\mathrm{m}$, enquanto o aumento do custo de ajuste aumenta o valor ótimo de m quando o nível do custo de defeituoso (retrabalho) é baixo (-) e diminui ou se mantém constante quando o nível do custo de defeituoso (retrabalho) está no nível alto (+). Verificamos também que o aumento do custo de defeituoso (retrabalho) diminui o valor ótimo de m.

No ponto onde o nível de ajuste é alto e os níveis do custo de defeituoso e de inspeção são baixos o valor ótimo de m é igual a $\mathrm{T}$, isto é, quando o custo de ajuste é muito maior que o custo de inspeção e o custo de defeituoso o melhor procedimento é a produção, sem interrupção, de $\mathrm{T}$ itens, inspecionar o último item produzido e retrabalhar os itens defeituosos que forem encontrados sem a necessidade de nenhum ajuste no processo. 


\section{Capítulo 3}

Neste capítulo, estudaremos a questão do monitoramento da qualidade na linha através de procedimentos Taguchi para processos de produção finita em que os itens defeituosos são refugados e substituídos. Mostraremos a dificuldade de se obter, neste caso, através de modelos estocásticos, uma expressão exata e explícita para o custo total do procedimento, em função do intervalo de inspeção, que facilite a busca do intervalo ótimo. Mostraremos, entretanto, através de um modelo de simulação, que o intervalo ótimo de inspeção existe e que podemos obter um valor aproximado para o mesmo, através da mesma técnica.

\subsection{Descrição do problema}

Um processo produz itens um a um em sequiência e, em condição de controle, todos os itens produzidos são conformes. Fora de controle, entretanto, todos os itens produzidos são não conformes e não admitem retrabalho.

Para monitorar a estabilidade da fração defeituosa do processo e identificar os itens não conformes para refugo, um procedimento Taguchi idêntico ao utilizado no Capítulo 2 é implementado. Devido ao refugo, o processo deverá permanecer ativo até que um lote com $\mathrm{T}$ itens conformes seja produzido. Esta particularidade caracteriza uma operação em horizonte aleatório e não propriamente finito.

Assim como no Capítulo 2, supomos que a inspeção de um item e a correção do processo são instantâneas, e que o número de itens produzidos até a mudança do estado de controle para o estado fora de controle tem distribuição geométrica com parâmetro $p$. A Figura 3.1 apresenta um ciclo típico de produção, caracterizado por uma seqüência (de comprimento geométrico) de itens conformes e seguida de uma sequiência de itens não conformes, interrompida pelo procedimento de inspeção. 


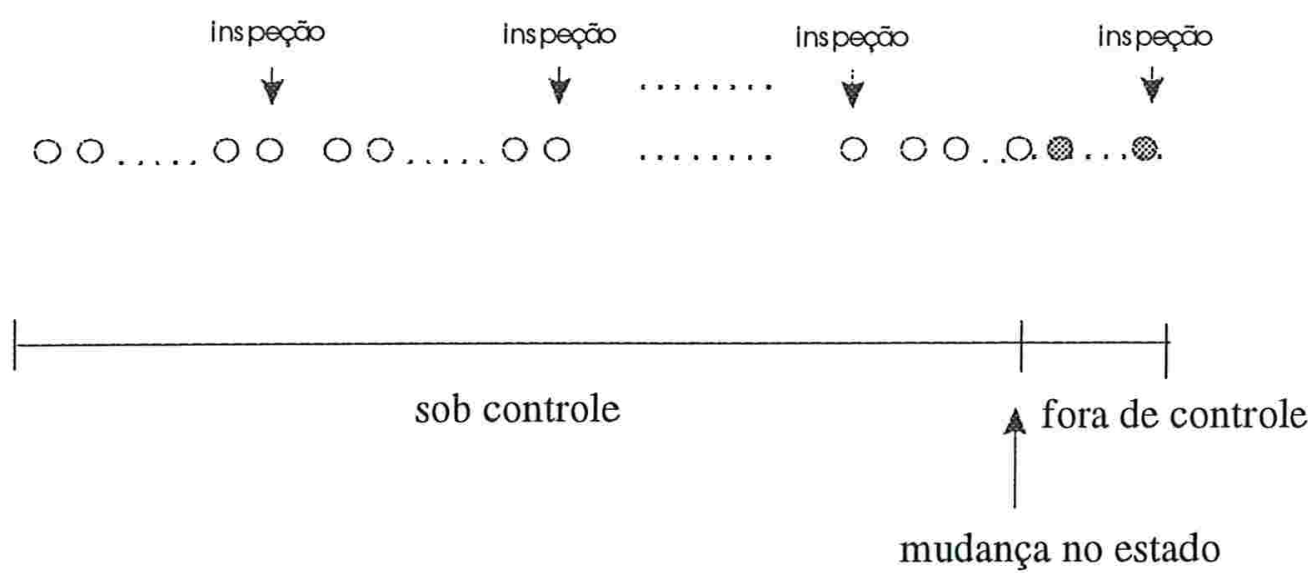

Figura 3.1 Esquema da mudança no estado do processo de produção.

Neste ponto o processo é reajustado e volta a operar sob o mesmo esquema até completar o lote. O intervalo de inspeção, entretanto, irá variar. Ele será igual a $m$ se a quantidade de itens conformes a produzir for maior que $m$. Caso contrário, esse intervalo será igual à quantidade de itens conformes que faltam produzir para completar $\mathrm{T}$.

Se não houver perda de controle, a quantidade de itens não conformes produzidos será igual a zero e o número de itens conformes produzidos será igual a T. Caso ocorram perdas de controle, o número de itens produzidos será maior que $\mathrm{T}$.

\subsection{Modelagem}

Para modelar estocasticamente este processo, podemos adotar dois caminhos. Um, não markoviano, em que partimos de blocos (conjuntos) independentes de unidades produzidas entre ajustes sucessivos, e outro, markoviano, em que partimos de blocos dependentes de unidades produzidas entre inspeções sucessivas. Desenvolveremos com detalhes, entretanto, apenas o primeiro caminho.

Considere, portanto, uma seqüência $\left\{W_{i} ; \mathrm{i} \geq 1\right\}$ de variáveis aleatórias independentes com distribuição geométrica, com parâmetro p. Mais, precisamente,

$$
P\left(W_{i}=w_{i}\right)=q^{w_{i}} \cdot p \quad w_{i}=0,1,2,3, \ldots
$$


Não é difícil ver que o evento $A_{1}=\left\{W_{i} \geq \mathrm{T}\right\}$ representa a situação em que a produção do lote não requer paradas do processo para ajuste. Neste caso, temos apenas um bloco de itens em que:

- o tamanho do bloco $B_{1}=T$;

- o número de itens conformes $V_{1}=T$;

- o número de itens não conformes $D_{1}=0$;

- o número de inspeções programadas $\left.L_{1}=\right] \frac{T}{m}[$;

- o número de inspeções retrospectivas $Z_{1}=0$.

De maneira análoga, para $\mathrm{k}>1$ o evento

$$
A_{k}=\left\{W_{1}<T, \ldots, W_{1}+\ldots+W_{k-1}<T, W_{1}+\ldots+W_{k} \geq T\right\}
$$

representa a situação em que a produção do lote requer exatamente $k-1$ paradas do processo para ajuste e, neste caso, são formados $k$ blocos de itens e no bloco $k$, a parada ocorre quando completamos o número de itens conformes igual a T. Assim, se denotarmos por $N_{i}$, $1 \leq i \leq k$, o número de unidades conformes que faltam ser produzidas para completar o lote, imediatamente antes de iniciar a produção do bloco i. Por exemplo, para o bloco 1:

- Tamanho do bloco

$$
\begin{aligned}
& B_{1}=\left[\frac{W_{1}}{m}\right] \cdot m+\min \left\{m, N_{1}-\left[\frac{W_{1}}{m}\right] \cdot m\right\}=\min \left\{\left(\left[\frac{W_{1}}{m}\right]+1\right) \cdot m, N_{1}\right\} \\
& \text { onde: }\left[\frac{W_{1}}{m}\right] \text { é o maior inteiro menor ou igual a } W_{1} / m .
\end{aligned}
$$

- Número de itens conforme $V_{1}=W_{1}$

- Número de itens não conformes

$$
D_{1}=B_{1}-W_{1}=\min \left\{\left(\left[\frac{W_{1}}{m}\right]+1\right) \cdot m, N_{1}\right\}-W_{1}
$$


- $\quad$ Número de inspeções retrospectivas

$$
Z_{1}= \begin{cases}D_{1} & \text { se } W_{1}>\left[\frac{W_{1}}{m}\right] \cdot m \\ D_{1}-1 & \text { se } W_{1}=\left[\frac{W_{1}}{m}\right] \cdot m\end{cases}
$$

- Número de inspeções programadas:

$$
\left.L_{1}=\right] \frac{B_{1}}{m}[
$$

Generalizando para o bloco i, $2 \leq i \leq k-1$, teremos:

- Tamanho do bloco

$$
B_{i}=\left[\frac{W_{i}}{m}\right] \cdot m+\min \left\{m, N_{i}-\left[\frac{W_{i}}{m}\right] \cdot m\right\}=\min \left\{\left(\left[\frac{W_{i}}{m}\right]+1\right) \cdot m, N_{i}\right\}
$$

- Número de itens conforme $V_{i}=W_{i}$

- Número de itens não conformes

$$
D_{i}=B_{i}-W_{i}=\min \left\{\left(\left[\frac{W_{i}}{m}\right]+1\right) \cdot m, N_{i}\right\}-W_{i}
$$

- Número de inspeções retrospectivas

$$
Z_{i}= \begin{cases}D_{i} & \text { se } W_{i}>\left[\frac{W_{i}}{m}\right] \cdot m \\ D_{i}-1 & \text { se } W_{i}=\left[\frac{W_{i}}{m}\right] \cdot m\end{cases}
$$

- Número de inspeções programadas: $\left.L_{i}=\right] \frac{B_{i}}{m}[$

Finalmente, para o bloco $k$ (último bloco), teremos

- Tamanho do bloco $B_{k}=N_{k}$ 
- Número de itens conformes $V_{k}=N_{k}$

- Número de itens não conformes $D_{k}=0$

- Número de inspeções retrospectivas $Z_{k}=0$

- Número de inspeções $\left.L_{k}=\right] \frac{B_{k}}{m}[$

As variáveis $B_{i}, V_{i}, D_{i}, Z_{i}$ e $L_{i}$ introduzidas acima podem ser definidas para todo $\mathrm{i} \geq 1$, a partir da seqüência $\left\{W_{i} ; \mathrm{i} \geq 1\right\}$. Para isto basta definir a seqüência $\left\{N_{i} ; \mathrm{i} \geq 1\right\}$ recursivamente, tornando-se: $N_{1}=T$ e $N_{i+1}=N_{i}-W_{i} \wedge N_{i}, i \geq 1$, onde $W_{i} \wedge N_{i}$ é o mínimo entre $W_{i}$ e $N_{i}$, e observar que:

$$
\begin{aligned}
& B_{i}=\min \left\{\mathrm{m} \cdot\left(\left[\frac{W_{i} \wedge N_{i}}{m}\right]+1\right) N_{i}\right\}, \\
& D_{i}=\min \left\{\left(\left[\frac{W_{i} \wedge N_{i}}{m}\right]+1\right) \cdot m, N_{i}\right\}-\left(W_{i} \wedge N_{i}\right), \\
& Z_{i}= \begin{cases}D_{i}-1 & \text { se } W_{i}=\left[\frac{W_{i}}{m}\right] \cdot m \text { e } W_{i}<N_{i} \\
\mathrm{D}_{\mathrm{i}} & \text { caso contrário }\end{cases} \\
& \mathrm{e} \\
& \left.L_{i}=\right] \frac{B_{i}}{m}[
\end{aligned}
$$

Com estas definições, podemos então obter uma expressão matemática do custo médio de produção de um lote com $\mathrm{T}$ itens conformes, $\mathrm{E}\left[\mathrm{C}_{\mathrm{T}}\right]$. Para tanto, vamos considerar os seguintes fatores de custo:

- o custo de inspeção ( $\left.\mathrm{C}_{\text {inspp }}\right)$

- o custo de ajuste $\left(\mathrm{C}_{\text {ajuste }}\right)$ 
- o custo de item defeituoso $\left(\mathrm{C}_{\text {def }}\right)$

Assim,

$$
E\left(C_{T}\right)=\sum_{k=1}^{\infty} E\left(C_{T} ; A_{k}\right)
$$

e não é difícil ver que para o caso em que não houver ajuste, o custo total é dado por:

$$
C_{T}=C_{\text {insp }} \cdot(] \frac{N_{1}}{m}[) \text { em } A_{I}
$$

e para $\mathrm{k}>1$, isto é, caso haja pelo menos um ajuste,

$$
\begin{aligned}
C_{T}=\sum_{\mathrm{i}=1}^{\mathrm{k}}\left[\mathrm{C}_{\text {insp }} \cdot\left(L_{i}+Z_{i}\right)+C_{\text {def }} \cdot D_{i}\right]+(k-1) \cdot C_{\text {ajuste }} \text { em } A_{k}, k>1 . \\
\quad \text { Assim, } E\left[C_{T}\right]=\sum_{k=1}^{\infty} \sum_{i=1}^{k} E\left[C_{\text {insp }}\left(L_{i}+Z_{i}\right)+C_{d e f} \cdot D_{i} ; A_{k}\right]+\sum_{k=1}^{\infty}(k-1) \cdot P\left(A_{k}\right) \cdot C_{\text {ajuste }}
\end{aligned}
$$

Se observarmos ainda que $\left\{A_{k} ; k \geq 1\right\}$ é uma partição do espaço amostral e que $N_{i}=0$ então $B_{j}=V_{j}=D_{j}=Z_{j}=L_{j}=0, \mathrm{j} \geq \mathrm{i}$, podemos expressar $\mathrm{E}\left[\mathrm{C}_{\mathrm{T}}\right]$ como

$$
E\left[C_{T}\right]=\sum_{i=1}^{\infty} C_{\text {insp }} \cdot E\left[L_{i} ; \mathrm{W}_{\mathrm{i}} \geq N_{i}\right]+\sum_{i=1}^{\infty} E\left[C_{\text {insp }} \cdot\left(L_{i}+Z_{i}\right)+C_{\text {def }} \cdot D_{i}+C_{\text {ajuste }} ; \mathrm{W}_{\mathrm{i}}<N_{i}\right]
$$

A obtenção de uma expressão fechada e explícita para $\mathrm{E}\left[\mathrm{C}_{\mathrm{T}}\right]$ em função de $m$, entretanto, esbarra na dificuldade de se obter os valores esperados da expressão (3.25). A obtenção de um valor de $m$ próximo do valor ótimo pode, todavia, ser feito através de um modelo de simulação. 


\subsection{Modelo de Simulação}

Desenvolveu-se um programa de computador a fim de simular o processo e encontrar o intervalo ótimo, aproximado, de inspeção. Neste programa, simula-se o processo de geração de $T$ itens conformes várias vezes (por exemplo: 5000 vezes) para cada m. Dessas simulações podemos obter o número médio de inspeções (Med_Insp), de ajustes (Med_Ajuste) e de defeituosos (Med_Def) para o cálculo do custo total médio para cada valor de $m$ ( 1 a $T)$.

Os itens são gerados um a um através de um gerador de dados Bernoulli com parâmetro $p$, onde 0 representa item conforme e 1 item não conforme. As variáveis utilizadas neste programa são:

- Número de itens conformes a ser produzido $(T)$

- Número de itens conformes produzidos no início do próximo intervalo entre inspeções $(V)$

- Intervalo entre inspeções, em itens ( $m$ ou $T-V$ )

- número de itens defeituosos na produção de T itens conformes ( Def)

- número de inspeções na produção de T itens conformes (insp)

- número de inspeções retrospectivas na produção de T itens conformes ( Retro)

- número de parada para ajuste na produção de T itens conformes (parada)

- Número de repetições da produção de T itens conformes para cada $m$ (intera)

- Valor gerado pela distribuição Bernoulli (item)

- número de itens conformes durante a simulação de um intervalo de inspeções (nitem)

os cálculos do número médio de ajuste (Med_Ajuste), de não conformes (Med_Def) e de inspeções (Med_Insp) para cada m são dados por:

Med_Ajuste $=$ parada $/$ intera 


$$
\begin{aligned}
& \text { Med_Def }=\text { Def } / \text { intera } \\
& \text { Med_Insp }=\text { insp } / \text { intera }
\end{aligned}
$$

O valor médio do custo total para cada $m$ são arquivados no vetor (vetorCusto). Dessa forma, o valor de $m$ correspondente ao mínimo dos valores do custo total médio, isto é, $\min \{$ vetorCusto $\}$, será o valor ótimo do intervalo entre inspeções. O fluxograma desse programa está na Figura 3.2 e o programa desenvolvido em S-Plus no Anexo 3. 


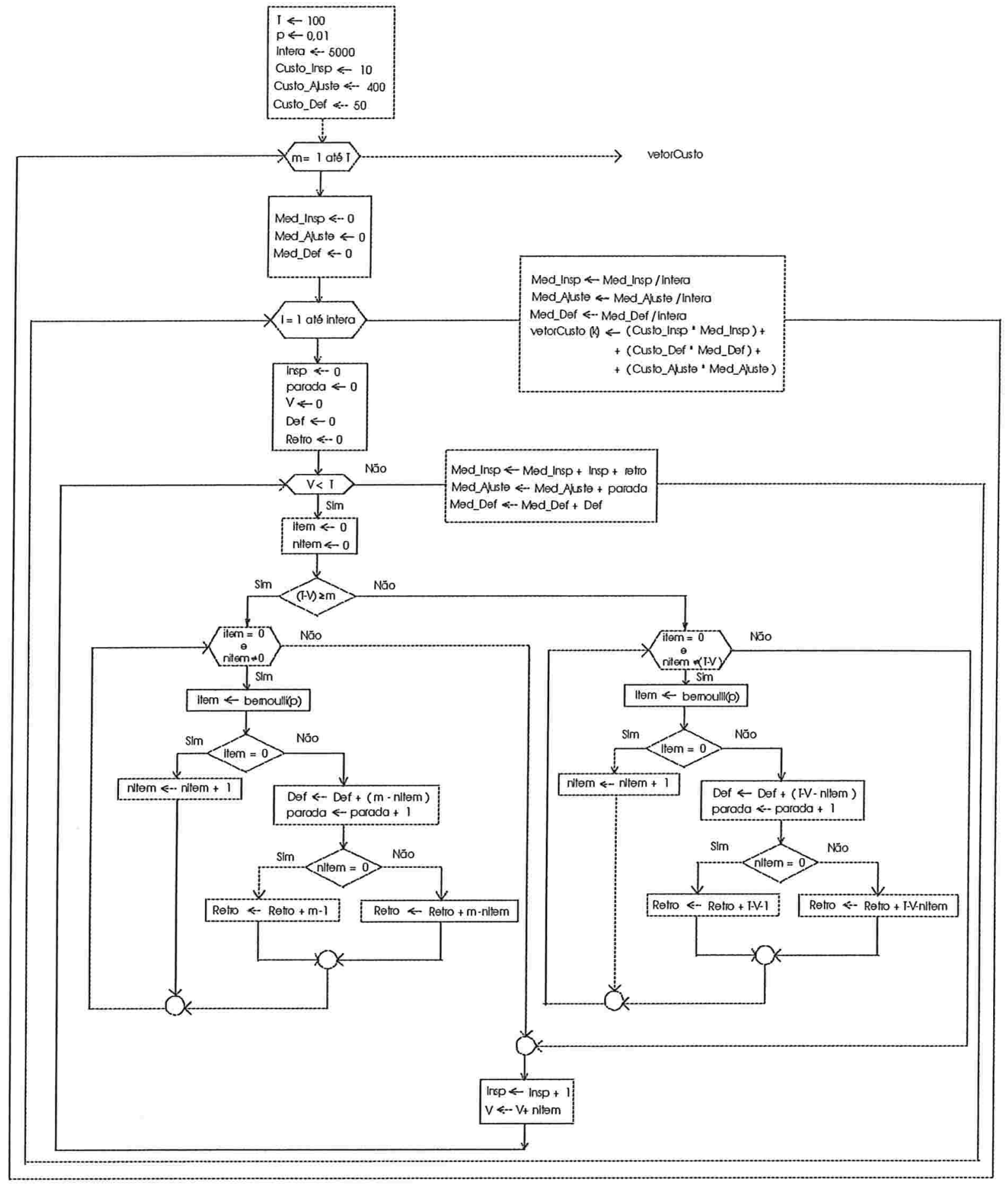

Figura 3.2 Fluxograma do simulador do processo de monitoramento Taguchi para horizonte finito com defeituosos como itens refugados. 


\subsection{Exemplo Numérico}

Os gráficos da Figura 3.3 e a Tabela 3.1 mostram o resultado da simulação quando adotamos os seguintes valores

- $\mathrm{T}=100$

- $\mathrm{p}=0.1 ; \mathrm{p}=0,01 ; \mathrm{p}=0,001 \mathrm{e} \mathrm{p}=0,0001$

- Custo de Inspeção = 10

- Custo de Ajuste $=400$

- Custo de Defeito $=50$

Os gráficos da Figura 3.3 mostram o comportamento do custo esperado total em função de m e de p.
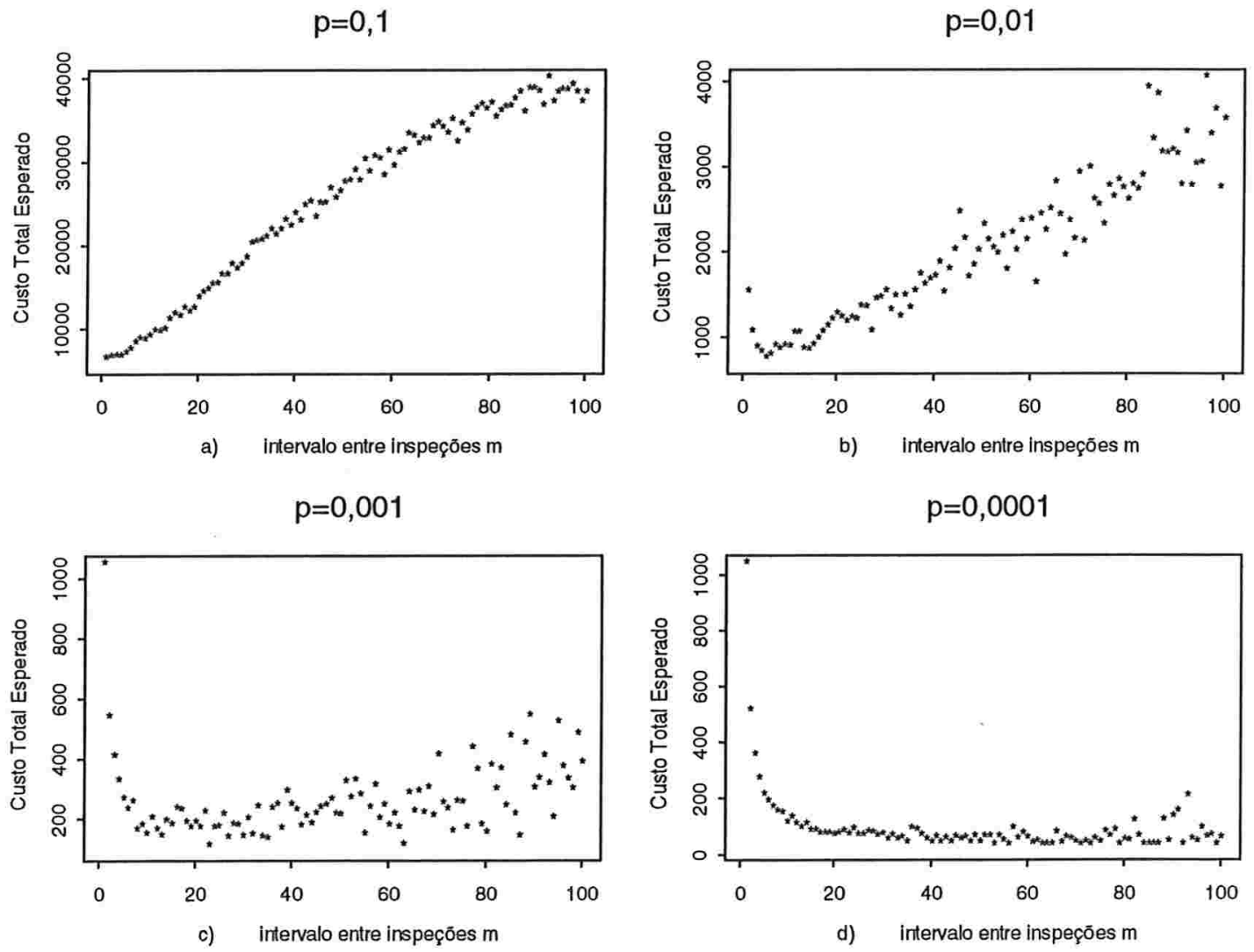

Figura 3.3 Gráficos do custo total esperado em função de m para a) $\mathrm{p}=0,1$; b) $\mathrm{p}=0,01$; c) $\mathrm{p}=0,001 \mathrm{e}$ d) $\mathrm{p}=0,0001$. 
Os valores ótimos, aproximados, de $m$ estão na Tabela 3.1.

Tabela 3.1: Tabela do valor de m mínimo para vários valores de $\mathrm{p}$.

\begin{tabular}{|c|c|c|}
\hline Caso & $\mathrm{P}$ & $\mathrm{m}$ ótimo \\
\hline 1 & 0,1 & 2 \\
\hline 2 & 0,01 & 6 \\
\hline 3 & 0,001 & 18 \\
\hline 4 & 0,0001 & 100 \\
\hline
\end{tabular}

Podemos verificar através dos gráficos que a dispersão dos pontos cresce em função de $m$. Isso ocorre principalmente porque o número de combinações de eventos possíveis aumenta com $m$. Podemos verificar através da Tabela 3.1 e dos gráficos da Figura 3.3, que o intervalo ótimo de amostragem aumenta quando diminuímos o valor de p. Observe que quando p é muito baixo ( 0,0001) a função parece decrescente, sem ponto de mínimo local e, portanto, o valor ótimo de $\mathrm{m}$ será $\mathrm{m}=\mathrm{T}$. 


\section{Capítulo 4}

\subsection{Conclusões}

Neste trabalho estudou-se dois problemas do procedimento Taguchi "On-Line" para atributos para processos de produção em pequeno lote. O primeiro, tratado no Capítulo 2 , os itens defeituosos são retrabalhados e corrigidos. Deste problema, obtivemos uma expressão que possibilita o cálculo do valor ótimo da frequiência de amostragem, m, minimizando o custo de monitoramento do processo. Dessa expressão verificamos que em duas situações a frequiência de amostragem ótima é igual ao tamanho do lote (T). Na primeira, bastante intuitivo, ocorre quando p é muito baixo. Na segunda, quando o custo de ajuste é muito maior que os custos de inspeção e de defeituoso. Analisando o efeito dos custos, concluímos que o aumento do custo de inspeção tende a diminuir o valor ótimo de $\mathrm{m}$, enquanto o aumento do custo de ajuste tende a aumentar o valor ótimo de $\mathrm{m}$ quando o nível do custo de defeituoso é baixo e tende a diminuir ou se manter constante quando o custo de defeituoso é alto. Verificamos também que o aumento do custo de defeituoso tende a diminuir o valor ótimo de $\mathrm{m}$.

No segundo problema, tratado no Capítulo 3, os itens defeituosos são refugados devido a dificuldade ou impossibilidade do retrabalho desse item. Para a solução desse problema desenvolveu-se um modelo de simulação para a obtenção do valor ótimo da frequiência de amostragem devido à grande dificuldade na modelagem analítica do custo esperado total do procedimento. Verificamos, através da simulação, que o valor ótimo da freqüência de amostragem tende a aumentar quando diminuímos o valor da probabilidade do processo sair de controle. 


\subsection{Sugestões de Pesquisa Futura}

Algumas sugestões para pesquisa futura são:

1. Estudo dos mesmos procedimentos (retrabalho e refugo dos itens não confomes) para o caso 2 apresentado por Taguchi et al (1989), isto é, quando o processo está fora de controle os itens produzidos terão fração de não conformes.

2. Análise com erro no diagnóstico.

3. Análise do procedimento Taguchi "On-Line" para atributos em horizonte finito com fração de itens não conformes retrabalhados e fração de itens não conforme refugados. 


\section{Bibliografia}

1. Banks, J. (1989), "Principles of Quality Control", John Wiley \& Sons, New York.

2. Benton, W.C. (1991), "Statistical process control and Taguchi method: a comparative evaluation" , Int. J. Prod. Res., 29, 1761-1770.

3. Borges, S.W., Ho,L.L., Tunes, O. (2000), "An anlysis of Taguchi's on-line quality monitoring procedure for attributes with diagnosis errors", RT-MAE 2000-2, São Paulo, SP.

4. Box, G.E.P., Luceño, A. (1997), "Statistical Control by Monitoring and Feedback Adjustment", John Wiley \& Sons, NY.

5. Carlyle, M.W., Montgomery, D.C., Runger, G.C.(2000), "Optimization Problems and Methods in Quality Control and Improvement", Journal of Quality Technology, 32 (1), 1-17.

6. Costa, A.F.B. (1998), "Gráficos de Controle $\bar{X}$ Para Processos Robustos", Gestão \& Produção, 5, 259-271.

7. Dehnad, K. (1989), "Quality Control, Robust Design, and Taguchi Method", Wadsworth \& Brooks/Cole.

8. Duncan, A.J. (1978), "The Economic Design of p-Charts to Maintain Current of a Process: Some Numerical Results", Technometrics, 20, 235-243.

9. Duncan, A.J. (1986), "Quality Control and Industrial Statistics”, Richard D. Irwin, Inc..

10. Gibra, I.N. (1978), "Economically Optimal Determination of the Parameters of $n p$ Control Charts", Journal of Quality Technology, 10, 12-19.

11. Ho, C.; Case, K.E. (1994), “Economic design of control-charts: a literature review for 1981-1991”. Journal of Quality Technology, 26), 39-53.

12. Hoel, P.G.; Port, S.C.; Stone, C.J. (1972), "Introduction to Stochastic Process", Houghton Mifflin Company.

13. Ladany, S.P. (1973), "Optimal use of control charts for controlling current production”, Management Science, 19, 763-772.

14. Montgomery, D.C., Heikes, R.G., Mance, J.F. (1975), "Economic Design of Fraction Defective Control Charts", Management Science, 21, 1272-1284. 
15. Montgomery, D.C. (1982), "The Economic Design of Control Chart: A Review and Literature Survey", Journal of Quality Technology, 40-43.

16. Montgomery, D.C. (2000), "Introduction to statistical quality control", John Wiley \& Sons, New York.

17. Nayebpour, M.R.; Woodall, W.H. (1993), “An Analysis of Taguchi's on-line quality monitoring procedures for attributes", Technometrics, 35, 53-60.

18. Ramos, A.W. (1995), "Controle Estatístico de Processo para Pequenos Lotes", Editora Edgard Blücher Ltda. São Paulo, SP.

19. Ryan, T. P. (1989), "Statistical Methods for Quality Improvement", John Wiley \& Sons, New York..

20. Ross, S.M. (1983), “Stochastic Process”, John Wiley \& Sons, New York.

21. Ross, S.M. (1997), “Simulation”, Academic Press.

22. Saniga, Erwin M. Economic Statistical Control-Chart Designs With an Application to $X$ and $R$ Charts, Technometrics, 31(3): 313-320, 1989.

23. Silva, P.A.L. (1994), "Técnicas Taguchi Para a Melhoria da Qualidade", $11^{\circ}$ SINAPE, Belo Horizonte-MG.

24. Sullivan, L.P. (1987), “The Power of Taguchi Methods", Quality Progress, 76-79.

25. Taguchi, G. (1981), "On-line Quality Control During Production”, Japanese Standars Association, Tokyo.

26. Taguchi, G. (1984), "Quality Evaluation for Quality Assurance", American Supplier Institute, Dearborn, MI.

27. Taguchi, G. (1985), "Quality Engineering in Japan", Communications in Statistics Theory and Methods, 14, 2785-2801.

28. Taguchi, G. (1996), "Introduction to Quality Engineering", Tokyo. Asian Productivity Association.

29. Taguchi, G.; Elsayed, E.A.; Hsiang, T.C. (1989), "Quality Engineering in Production Systems", McGraw-Hill, New York, N.Y.

30. Taguchi, G.; Elsayed, E.A.; Hsiang, T.C. (1990), "Engenharia de Qualidade em Sistemas de Produção”, McGraw Hill, São Paulo, SP. 
31. Turnes, O. (1997), "Custo da Qualidade: Planejamento Econômico dos Gráficos de Controle por Atributos e Modelos Correlatos", Tese de doutorado, Florianópolis, SC.

32. Venables, W.N., Ripley, B.D. (1997), "Modern Applied Statistics With S-Plus", Spreiger-Verlog, NY.

33. Williams, W.W., Looney, S.W., Peters, M.H. (1985), “Use of Curtailed Sampling Plans in the Economic Design of np Control Charts", Technometrics, 27, 57-63.

34. Woodall, W.H., Montgomery, D.C. (1999), "Research Issues and Ideas in Statistical Process Control”. Journal of Quality Technology, 31, 376-386. 


\section{Anexo 1}

Programa em S-Plus para obter o gráfico e o valor de $\mathrm{m}$ que minimiza o custo esperado total.

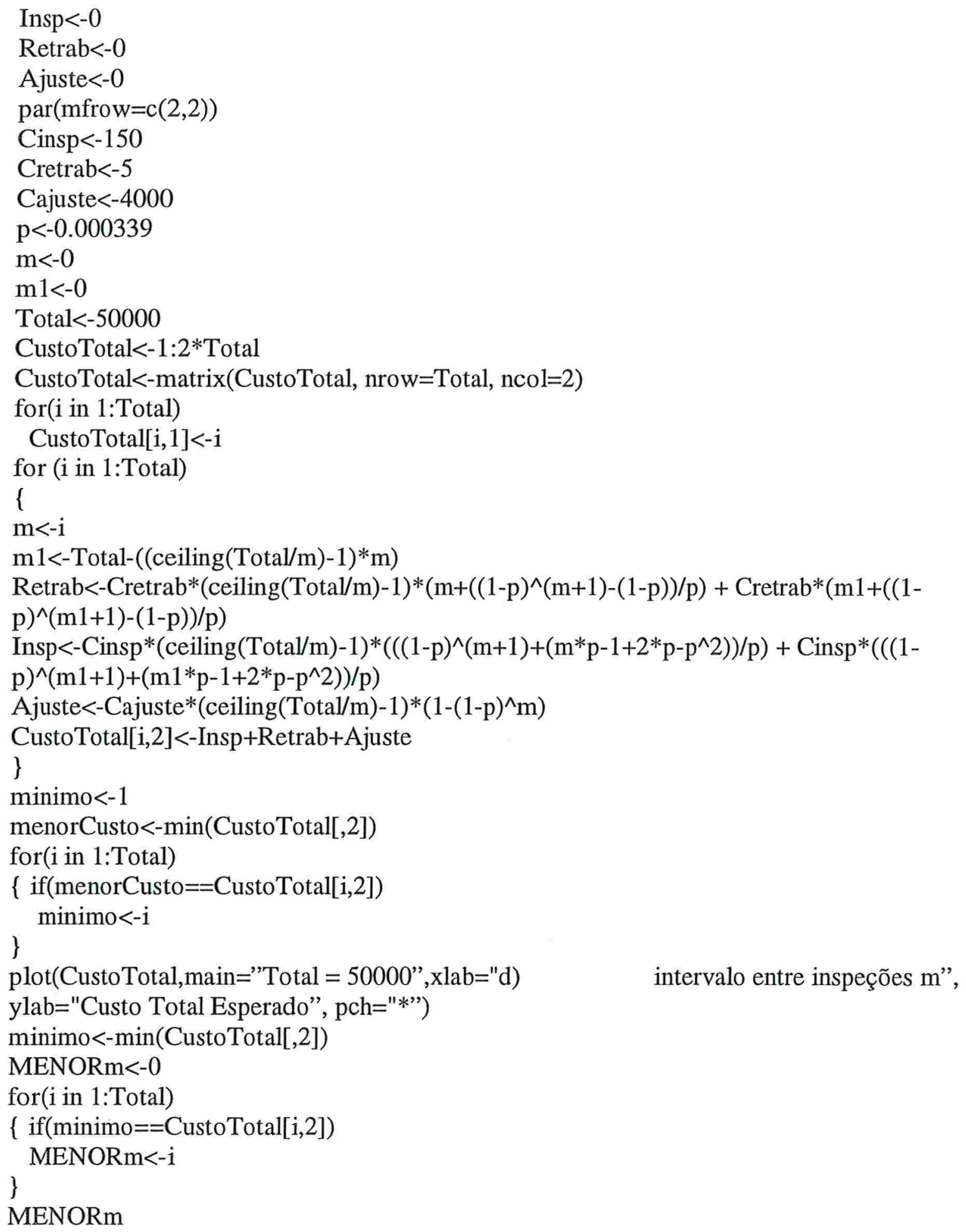




\section{Anexo 2}

Programa em S-Plus para obtenção do m para minimizar o custo esperado total para vários valores de $\mathrm{p}$.

Insp $<-0$

Retrab $<-0$

Ajuste $<-0$

$\operatorname{par}(\operatorname{mfrow}=\mathrm{c}(1,1))$

Cinsp $<-10$

Cretrab $<-50$

Cajuste $<-400$

ind $<-0$

auxiliar $<-1$

Vetorpm $<-1: 50$

Vetorpm<-matrix (Vetorpm, nrow $=25, \mathrm{ncol}=2$ )

Vetorpm[1,1]<-0.000001; Vetorpm[2,1]<-0.00001; Vetorpm[3,1]<-0.00005;

Vetorpm $[4,1]<-0.00009$;

Vetorpm[5,1]<-0.0001; Vetorpm[6,1]<-0.0003; Vetorpm[7,1]<-.0007; Vetorpm[8,1]<-

0.001 ;

Vetorpm[9,1]<-0.003; Vetorpm[10,1]<-0.01; Vetorpm[11,1]<-0.03; Vetorpm[12,1]<-0.05;

Vetorpm[13,1]<-0.09; Vetorpm[14,1]<-0.1; Vetorpm[15,1]<-0.105; Vetorpm[16,1]<-0.11;

Vetorpm $[17,1]<-0.115$; Vetorpm[18,1]<-0.118; Vetorpm[19,1]<-0.119; Vetorpm[20,1]<0.11903 ;

Vetorpm[21,1]<-0.119032; Vetorpm[22,1]<-0.119036; Vetorpm[23,1]<-0.119037;

Vetorpm[24,1]<-0.3; Vetorpm[25,1]<-0.50

for( $\mathrm{k}$ in 1:25)

\{

$\mathrm{p}<-$ Vetorpm[k,1]

$\mathrm{m}<-0$

$\mathrm{m} 1<-0$

Total $<-500$

CustoTotal<-1:2*Total

CustoTotal<-matrix(CustoTotal, nrow=Total, ncol=2)

for(i in 1:Total)

CustoTotal $[\mathrm{i}, 1]<-\mathrm{i}$

for (i in 1:Total)

\{

$\mathrm{m}<-\mathrm{i}$

m1<-Total-((ceiling(Total/m)-1)*m)

Insp<-Cinsp*(ceiling $($ Total/m) -1$) *\left(\mathrm{~m}+\left((1-\mathrm{p})^{\wedge}(\mathrm{m}+1)-(1-\mathrm{p})\right) / \mathrm{p}\right)+\operatorname{Cinsp}^{*}(\mathrm{~m} 1+((1-$

$\left.\left.\mathrm{p})^{\wedge}(\mathrm{m} 1+1)-(1-\mathrm{p})\right) / \mathrm{p}\right)$

Retrab $<-\operatorname{Cretrab}^{*}(\text { ceiling }(\text { Total } / \mathrm{m})-1)^{*}\left(\left((1-\mathrm{p})^{\wedge}(\mathrm{m}+1)+\left(\mathrm{m}^{*} \mathrm{p}-1+2 * \mathrm{p}-\mathrm{p}^{\wedge} 2\right)\right) / \mathrm{p}\right)+$

$\operatorname{Cretrab}^{*}\left(\left((1-\mathrm{p})^{\wedge}(\mathrm{m} 1+1)+\left(\mathrm{m} 1^{*} \mathrm{p}-1+2 * \mathrm{p}-\mathrm{p}^{\wedge} 2\right)\right) / \mathrm{p}\right)$

Ajuste $<-$ Cajuste* $(\text { ceiling }(\text { Total } / \mathrm{m})-1)^{*}\left(1-(1-\mathrm{p})^{\wedge} \mathrm{m}\right)$

CustoTotal[i,2]<-Insp+Retrab+Ajuste 
minimo $<-1$

menorCusto $<-\min$ (CustoTotal[,2])

for(i in 1:Total)

\{

if $($ menorCusto $==$ CustoTotal $[i, 2])$

minimo $<-\mathbf{i}$

\}

Vetorpm $[\mathrm{k}, 2]<-$ minimo

\}

plot(Vetorpm[,1],Vetorpm[,2], main="Gráfico de m(minimo) x p",xlab="p", ylab="m mínimo", pch="*")

Vetorpm 


\section{Anexo 3}

Programa em S-Plus para obter o gráfico e o m que minimiza o custo esperado total para procedimento Taguchi em horizonte finito e quando os itens defeituosos são refugados.

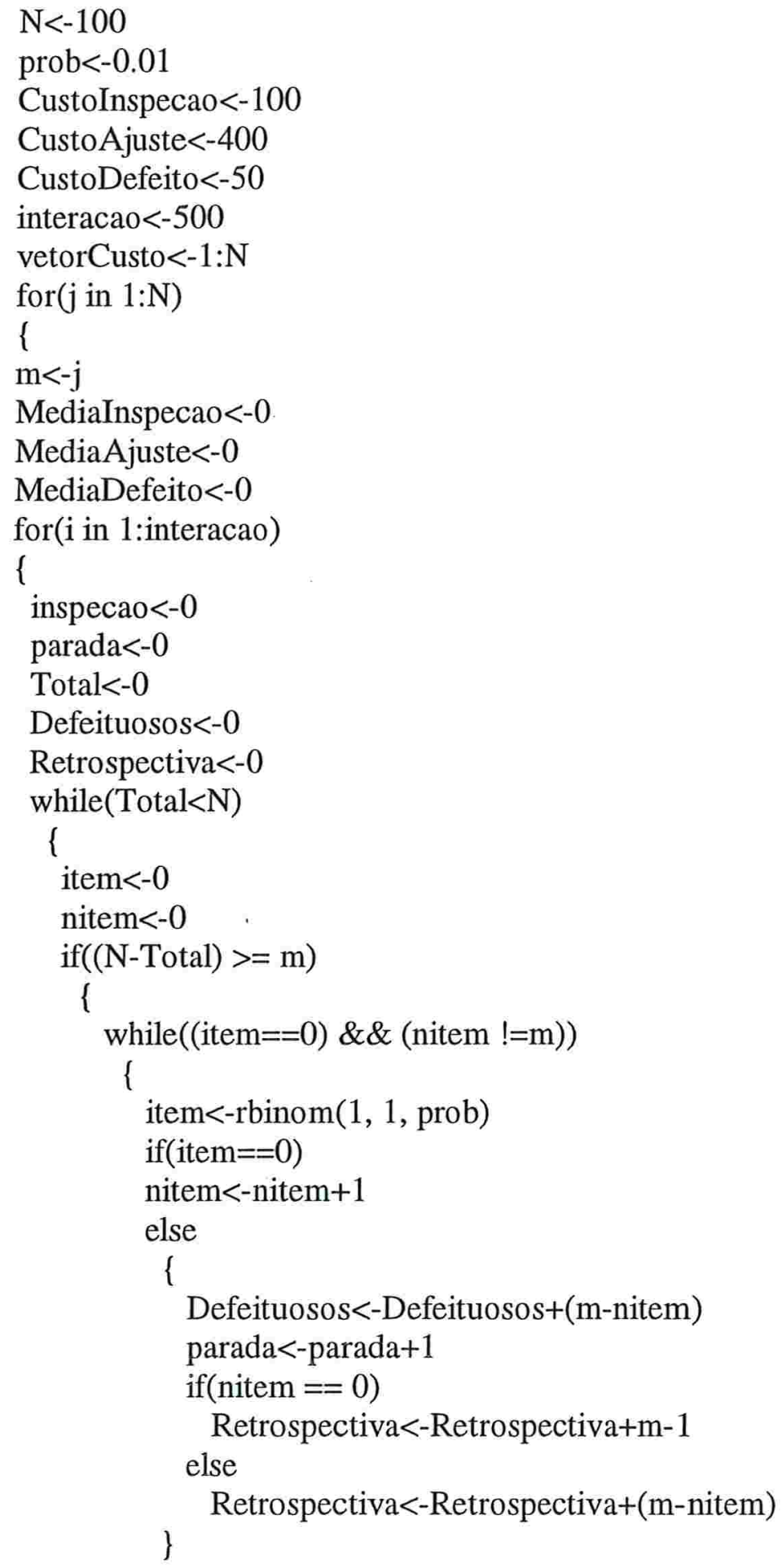




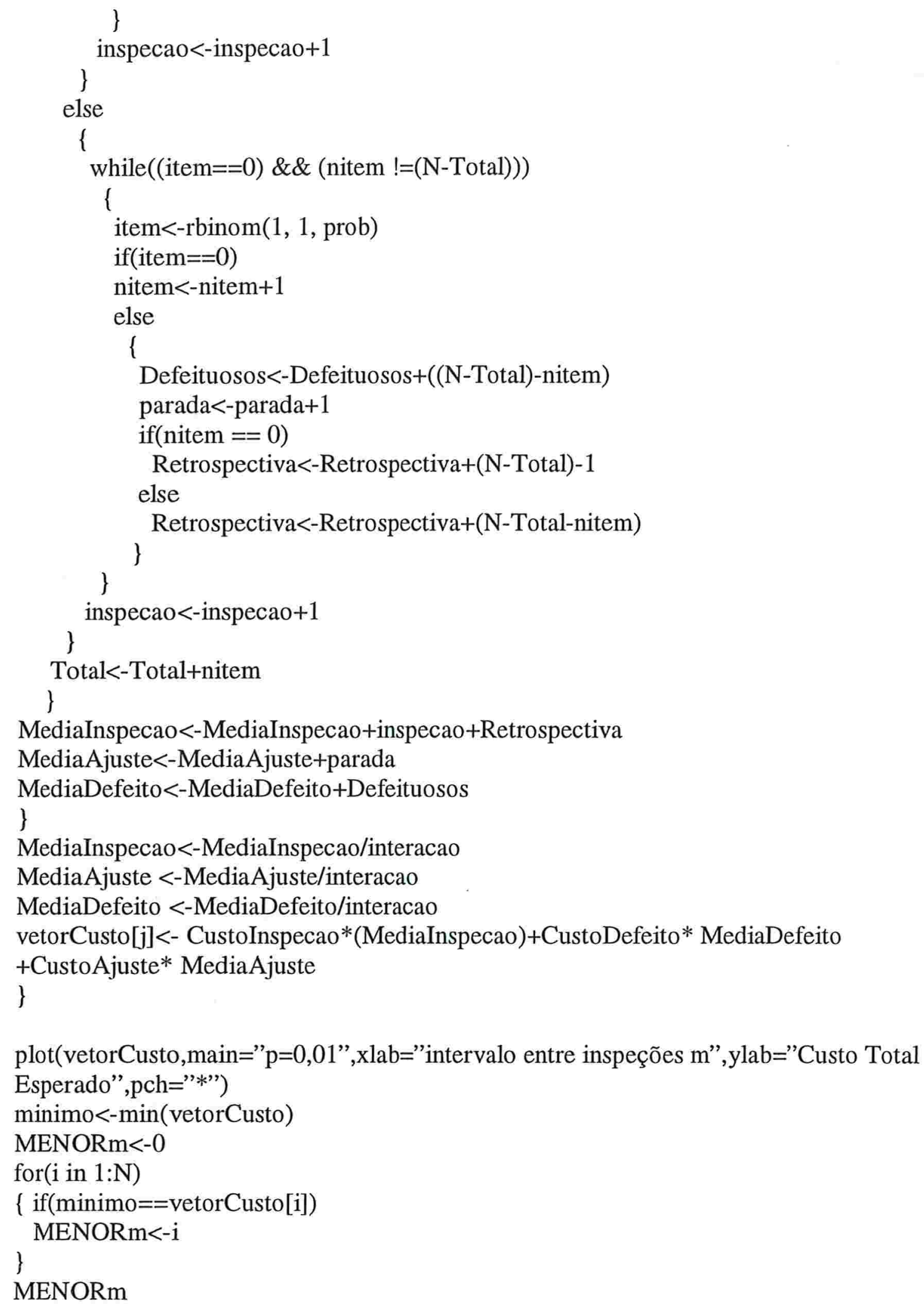

